\title{
PROBABILIDAD DE CORRECCIÓN SÚBITA DE CUENTA CORRIENTE PARA COSTA RICA: UN ENFOQUE DE ANÁLISIS DE SUPERVIVENCIA
}

\author{
Jorge León Murillo ${ }^{1}$ \\ Esteban Méndez-Chacón²
}

\begin{abstract}
RESUMEN
Mediante un modelo de análisis de supervivencia con datos de panel, se realiza un análisis exploratorio para la estimación de la probabilidad de correcciones súbitas en la cuenta corriente costarricense. Los resultados obtenidos muestran que la probabilidad de reversión de cuenta corriente disminuye cuando: i) se incrementa el crecimiento real para los siguientes cinco años; ii) mejora la situación externa de países considerados similares; iii) aumenta la tasa de dependencia económica; iv) el porcentaje del PIB mundial con el que se han ratificado acuerdos comerciales se incrementa; v) la acumulación de reservas monetarias se acelera; $y$ vi) el marco institucional se vuelve más democrático. Por otra parte, una mayor productividad total de los factores en los cinco años siguientes incrementa la probabilidad de reversión de cuenta corriente. El efecto del control de capitales sobre la probabilidad mostró un comportamiento ambiguo. El análisis se realiza para 116 países con datos anuales de 1981 a 2012.
\end{abstract}

PALABRAS CLAVE: MODELOS DE SUPERVIVENCIA, BALANCE EXTERNO, MERCADOS EMERGENTES.

\section{ABSTRACT}

Using a survival model approach using data panel, an exploratory analysis is made to estimate the probability of a sudden current account correction in Costa Rica. The results show that the estimated probability of a current account reversal decreases when: i) the five years ahead real-growth increases, ii) the external situation of similar countries improves, iii) the economic dependency ratio increases, iv) the world's GDP percentage for which the country have signed a trade agreement increases, $v$ ) reserves accumulation accelerates and vi) the institutional framework becomes more democratic. On the other hand, an increase in the total factor productivity (TFP) growth 5-year ahead raises likelihood of a reversal. The effect of capital controls on the probability showed an ambiguous behavior. Annual data from 1981 to 2012 for 116 countries is used.

KEY WORDS: SURVIVAL MODEL, EXTERNAL BALANCE, EMERGING MARKET. 


\section{INTRODUCCIÓN}

Costa Rica es una economía pequeña y abierta al comercio de bienes y servicios, así como al flujo de capitales. En 2014, el déficit de cuenta corriente del país se ubicó en un 5,18\% del PIB, financiado casi en su totalidad con ingresos de capital de largo plazo, principalmente inversión extranjera directa ${ }^{3}$. Este patrón de déficit $y$ financiamiento se ha mantenido relativamente estable durante la última década, en donde el país ha presentado un déficit estructural de cuenta corriente de alrededor de un 5\% del PIB ${ }^{4}$. Si bien en apariencia para el caso costarricense es factible mantener déficit de cuenta corriente estructural siempre existe la posibilidad que choques externos o internos hagan imposible sostener estos niveles de déficit, por lo cual acarree una corrección en la cuenta corriente del país.

La evidencia empírica sugiere que las correcciones súbitas ${ }^{5}$ en el déficit de cuenta corriente llevan aparejadas recesiones, quiebras y contracción del crédito interno. (Calvo, 1998). Por lo tanto, la estabilidad macroeconómica de un país como Costa Rica depende en gran medida de la evolución del comercio internacional $y$ de los flujos de capitales.

El presente documento estima la probabilidad de correcciones en la cuenta corriente para el caso de la economía costarricense. El modelo sirve para analizar el riesgo de una corrección súbita en el corto o mediano plazo. Para obtener un estimado de esta probabilidad se utiliza la metodología de modelos de supervivencia, los cuales describen el tiempo en un estado determinado antes de la transición a otro estado; en este caso particular, el tiempo que permanece un país en déficit antes de experimentar una reversión de cuenta corriente.

El documento tiene un carácter exploratorio tanto a nivel nacional como internacional por el uso de la metodología de supervivencia, con datos de panel para correcciones súbitas de cuenta corriente. Esta metodología no ha sido utilizada con anterioridad para el caso costarricense; a su vez, el uso de estimaciones Probit y de análisis de duración con datos de panel para medir la probabilidad de correcciones súbitas provee de un análisis más robusto. Se provee de una herramienta robusta que permita medir el nivel de riesgo de una corrección súbita de cuenta corriente para Costa Rica, con el fin de tomar medidas correctivas a tiempo.

En la literatura relevante, no existe una clara definición de una corrección súbita de cuenta corriente $^{6}$. Para este análisis, se decidió definir dos criterios para identificar una reversión de cuenta corriente en el momento $t$ : el primero es una reducción en el déficit de cuenta corriente de al menos 2 puntos porcentuales del PIB; el segundo es una reducción de 5 puntos porcentuales del PIB. Debido a las escasas observaciones para Costa Rica, $y$ a que para el periodo 1981 al 2012 se presentaron pocos episodios de reversiones de cuenta corriente, se estima el modelo utilizando un panel no balanceado de 116 países para el caso de reversiones iguales o mayores a 2 puntos porcentuales y otro de 91 países para el caso de mayores a 5 puntos porcentuales.

Entre los resultados obtenidos, se encuentra que un incremento en el crecimiento real del PIB futuro a cinco años en relación al resto del mundo disminuye la probabilidad de una reversión, lo cual es coherente con el enfoque intertemporal de la cuenta corriente. Además, menores déficits de cuenta corriente de países considerados similares reducen la probabilidad de una reversión, por vía de una reducción en la probabilidad de contagio. Una posible explicación para este resultado es que si un grupo de países son clasificados por los inversionistas como similares entre sí, y alguno de

\footnotetext{
3 Monge y Torres (2009) muestran el comportamiento de la inversión extranjera directa y su capacidad para financiar el déficit de cuenta corriente de la balanza de pagos.

En el Anexo 1 se presenta la evolución histórica de la cuenta corriente de Costa Rica entre los años 2000 y 2014.

Una corrección súbita de cuenta corriente está asociada a lo que la literatura denomina crisis de balanza de pagos.

Entendiéndose esta como una reducción o eliminación abrupta del déficit de cuenta corriente del país, siendo esta corrección igual o mayor a dos puntos porcentuales del PIB.
} 
ellos se enfrenta a problemas con la sostenibilidad de su cuenta corriente, los inversionistas podrían negarse a prestarles a los demás países del grupo.

También se encuentra que incrementos en la tasa de dependencia (la relación entre la población mayor a 65 años y la que se encuentra entre los 15 a 64 años) reducen la probabilidad de una reversión. De acuerdo con la hipótesis del ciclo de vida el crecimiento en la población inactiva reduciría los ahorros, puesto que en la economía existirían agentes que consumen más de lo que producen. Esto implicaría un aumento en el déficit de cuenta corriente. Otro factor significativo al explicar la probabilidad de una corrección súbita de cuenta corriente es el porcentaje del PIB mundial con el cual el país ha ratificado acuerdos comerciales. Un incremento en ese porcentaje implica una reducción en las probabilidades de reversión de la cuenta corriente. Una posible explicación es que los acuerdos comerciales refuerzan los compromisos de un país a la apertura comercial, y con ello se reducen las dificultades para afrontar deudas con el exterior.

El presente estudio, además, presenta evidencia de que la acumulación de reservas internacionales contribuye a reducir la probabilidad de reversión de cuenta corriente. Finalmente, la evidencia muestra que la convergencia del marco institucional del país a un sistema democrático, disminuye la probabilidad de una corrección súbita de cuenta corriente. Este resultado puede deberse a la protección de derechos de propiedad que van correlacionados con un marco institucional favorable.

También se encuentra que un incremento en el crecimiento de la productividad total de los factores (PTF) a 5 años eleva la probabilidad de una reversión. Este resultado puede ser ejemplo de la paradoja de Feldstein-Horioka. Si los aumentos en la PTF están relacionados con mayores niveles de inversión -de acuerdo con la paradoja los ahorros nacionales- deberían incrementarse y, por lo tanto, el déficit de cuenta corriente disminuir. No obstante, este resultado requiere mayor investigación.

Los controles de capitales muestran un efecto ambiguo en la probabilidad de reversión de cuenta corriente. Para reversiones de al menos 2 puntos porcentuales del PIB la reducción de controles de capital disminuye la probabilidad de una reversión. No obstante, para reversiones de al menos 5 puntos porcentuales del PIB el efecto de una reducción en los controles de capital incrementa la probabilidad de una reversión. Vale destacar que en ningún caso el efecto es estadísticamente significativo.

Con los resultados es posible estimar la probabilidad de reversión o corrección súbita de cuenta corriente para Costa Rica. Se puede concluir que las reversiones que efectivamente sucedieron en el país están correlacionadas con incrementos en las probabilidades de reversión estimadas por el modelo. Las predicciones fuera de muestra se realizan para el periodo 2013-2017. Los resultados sugieren que la cuenta corriente de Costa Rica enfrenta pocas probabilidades de una reversión, incluso de al menos 2 puntos porcentuales del PIB. Sin embargo, es importante prestar atención a cambios no anticipados en las variables relevantes. Los efectos encontrados en las variables, así como las probabilidades de reversión estimadas se mantienen al realizar las estimaciones con un modelo de supervivencia que considere el hecho de que la información sobre duración se registra en variable discreta.

\section{Literatura relacionada}

En la literatura no existe un consenso en la definición de una corrección súbita de cuenta corriente, sin embargo existen algunos puntos en común entre los investigadores del tema. Ferretti y Razin (2000) consideran una reversión como una mejora grande y persistente en el balance de cuenta corriente, que satisface: 1) una reducción promedio en el déficit de cuenta corriente de al menos 3 puntos porcentuales del PIB (también se evalúa el caso de 5 puntos porcentuales del PIB) con respecto a los tres años anteriores al evento; 2) el déficit máximo después de la reversión no podría ser más grande que el déficit mínimo en los tres años anteriores a la reversión y 3) el déficit 
promedio de cuenta corriente debe reducirse en al menos un tercio. Estos criterios se diseñan para excluir reversiones temporales y ajustes parciales de déficit grandes.

Por su parte, Edwards (2004) define una reversión de cuenta corriente como una reducción del déficit de cuenta corriente de al menos 4\% del PIB en un año. A su vez Bugamelli y Paternò (2009) eligen una definición de reversión de cuenta corriente en el momento $t$ tal que: 1) el balance de cuenta corriente en el momento $t-1$ sea deficitario; 2 ) el balance de cuenta corriente deba mejorar en al menos 5 puntos porcentuales del PIB; y 3) el tamaño de la mejora deba exceder la mitad del balance de cuenta corriente en $t-1$.

En cuanto a la metodología empleada para la estimación y los resultados cabe destacar que, Ferretti y Razin (2000) basan sus estimaciones en un modelo probit multivariado, y encuentran que es más probable que ocurran reversiones en países con déficit persistente, bajos niveles de reservas internacionales y términos de intercambio desfavorables. Edwards (2004), utilizando un modelo probit, concluye que la probabilidad de reversión es mayor para países con déficit de cuenta corriente graves, un alto endeudamiento externo respecto al PIB, un rápido crecimiento de los préstamos domésticos, y un bajo crecimiento del PIB antes de la reversión. Por otra parte, la probabilidad de reversión es baja para países que tienen un alto nivel de reservas internacionales y un alto nivel de apertura comercial.

Por su parte Bugamelli y Paternò (2009) emplean un modelo probit multivariado, utilizando variables instrumentales para evaluar aspectos de causalidad. Los resultados indican que las remesas de los trabajadores disminuyen la probabilidad de reversión de cuenta corriente. Además de las remesas, un alto nivel de reservas internacionales y un bajo o nulo déficit de cuenta corriente inicial reducen la probabilidad de reversión.

En relación al estudio de reversión de cuenta corriente mediante modelos de supervivencia, Jauregui (2006) analiza la duración de superávits que siguen después de correcciones de cuenta corriente. Utiliza un Modelo Cox de Riesgo Proporcional y datos trimestrales del International Financial Statistics del Fondo Monetario Internacional (FMI) de 1980 a 2005. El inicio del evento viene dado por tres condiciones: 1) presión sobre el mercado cambiario; 2) el balance de cuenta corriente tiene que ser deficitario en el periodo anterior; y 3) tiene que presentarse una reducción del déficit de cuenta corriente de al menos un punto porcentual en un trimestre.

En el World Economic Outlook de abril 2007 (Fondo Monetario Internacional, 2007), se aplica el análisis de supervivencia para explicar el déficit de las economías desarrolladas, y el superávit de las emergentes y las exportadoras de petróleo. Los datos cubren el periodo 1960-2006 e incluyen los 47 países con los mayores PIB per cápita (en dólares internacionales) en 2004. Se define un desbalance como un episodio donde la cuenta corriente es mayor que el $2 \%$ del PIB por al menos 5 años, en donde no ha ocurrido ninguna reversión (Fondo Monetario Internacional, 2007). Similarmente, una reversión de cuenta corriente se define como un cambio en el balance de cuenta corriente de al menos 2,5 puntos porcentuales del PIB y al menos 50\% del balance inicial de cuenta corriente. Los resultados sugieren que para los países exportadores de petróleo, las reversiones de superávits se deben a caídas en los precios de materias primas. Además, en las economías desarrolladas la reversión de superávits se presenta con apreciaciones del tipo de cambio e incrementos en la demanda doméstica. En contraste, reversiones del déficit tienden a ser precedidas por una brecha del producto positiva.

Aplicando las definiciones anteriores, el World Economic Outlook de octubre 2008, (Fondo Monetario Internacional, 2008) utiliza modelos de supervivencia para explicar cómo desbalances en cuenta corriente en economías emergentes pueden ser sostenibles. Se utiliza información de 19602007 y el interés principal es en las economías emergentes europeas y países asiáticos. El análisis permite concluir que un déficit persistente no necesariamente termina abruptamente, lo cual podría reflejar el hecho de que dichos déficits pueden ser signo de prosperidad económica; por ejemplo debido a una abundancia de oportunidades de inversión o una convergencia en productividad. En general, 
los principales factores que permiten un déficit de cuenta corriente prolongado son una alta cantidad inicial de activos extranjeros, apertura de la cuenta de capital, y oportunidades de crecimiento favorables. Además, se encuentra que la situación de déficit en los países emergentes en Europa es más persistente que otras regiones. La rápida liberalización de mercados financieros domésticos y la apertura de cuentas de capital, que atraen grandes cantidades de capital y favorecen el surgimiento de bancos extranjeros, son probables causas de la menor probabilidad de presentarse una corrección de cuenta corriente en los mercados emergentes en Europa.

Kintanar (2009) utiliza modelos de supervivencia semi-paramétricos para analizar el intervalo de tiempo desde el inicio de un déficit de cuenta corriente a su reversión. La base de datos cubre 65 países de 1970 a 2007. La tasa de interés real, y la interacción entre tipo de cambio real y los niveles de reservas reducen la probabilidad de reversión, e incrementos en la tasa de depreciación, el crédito doméstico, altas tasas de inflación, un alto nivel de reservas a PIB y un incremento en el balance regional de cuenta corriente incrementan la probabilidad de reversión.

\section{METODOLOGÍA}

Esta sección describe el modelo de supervivencia empleado para la estimación y los datos empleados. El lector interesado puede encontrar más detalles en Lancaster (1992). La presente exposición se deriva de Cameron y Trivedi (2009).

\section{Datos}

Los datos utilizados son anuales y cubren de 1981 a 2012, además incluyen 116 países para el caso de reversiones de al menos 2 puntos porcentuales del PIB y 91 países para el caso de reversiones de al menos 5 puntos porcentuales del PIB (la lista de países incluidos se puede encontrar en el Anexo 1 y 2 respectivamente). El tamaño de muestra cambia según la magnitud de la reversión puesto que para tratar la censura a la izquierda hay que descartar observaciones, $y$ no todo país que ha enfrentado una reversión de al menos 2 puntos porcentuales ha experimentado una reversión de al menos 5 puntos porcentuales (considere por ejemplo a Austria, el Reino Unido o Italia).

El crecimiento del PIB real futuro a 5 años ${ }^{7}$ se obtiene del "World Economic Outlook" de octubre $2013^{8}$. Para las estimaciones se utiliza el crecimiento relativo a un promedio ponderado del crecimiento del PIB real mundial. Por lo tanto, se refiere al cambio porcentual del PIB real a 5 años menos el cambio porcentual del PIB mundial a 5 años. En las estimaciones se rezaga esta variable un año.

El crecimiento de la Productividad Total de los Factores (PTF) a 5 años se utiliza las estimaciones hechas por el Econmap del Centre d'Etudes Prospectives et d'Informations Internationales (CEPII). Se considera esta variable como proporción al crecimiento de la productividad mundial a 5 años y se rezaga un año en las estimaciones. La productividad mundial a 5 años se obtiene como un promedio ponderado del crecimiento de las productividades de cada país. La PTF es estimada por el CEPII basado en un modelo Nelson-Phelps. La manera en que se modela el crecimiento implica que este es mayor cuando los niveles de capital humano son mayores; la velocidad del crecimiento de PTF se reduce con la cercanía a la frontera de la PTF; y el efecto es mayor para altos niveles de capital humano (Fouré, Bénassy-Quéré y Fontagné, 2010).

La variable utilizada en el periodo t es el crecimiento en el periodo $t+5$, bajo el supuesto de previsión perfecta por parte de los agentes.

8 Con datos observados para años anteriores al 2013 y le proyección realizada por el WEO para años posteriores. 
La tasa de dependencia es la relación de la población mayor de 65 años entre la población entre 15 a 64 años. La información se obtiene del "US Census Bureau International Data Base".

Se utiliza el índice Chinn-Ito para estimar el grado de apertura de la cuenta de capitales. Este índice se basa en una variable dicotómica que codifica las tabulaciones de las restricciones a transacciones financieras reportadas en el Annual report on Exchange Arrangements and Exchange Restrictions (AREAER) del FMI. El índice asume valores mayores conforme más abierta es la economía a transacciones con el exterior (Chinn y Ito, 2008). En las estimaciones se rezaga esta variable un año.

Se construye la variable cuenta corriente regional, como un promedio ponderado de los balances de cuenta corriente de los países localizados en la misma área geográfica. La delimitación de las áreas geográficas se basa en la distribución realizada por la Conferencia de las Naciones Unidas sobre Comercio y Desarrollo. En las estimaciones, la variable está rezagada un periodo. Las regiones utilizadas se muestran en el Anexo 3.

Utilizando la información sobre acuerdos comerciales producida por De Sousa (2012), se construye una nueva variable que se denominará acuerdos comerciales. Esta consiste en un promedio ponderado del PIB mundial con el que se tiene ratificados tratados comerciales. Cuando su valor se incrementa, es mayor el porcentaje del PIB mundial con el que el país posee acuerdos comerciales. En las estimaciones se rezaga esta variable un año.

Los cambios en las reservas son las variaciones en las reservas internacionales en relación al cambio presentado a nivel mundial. Se incluye en las estimaciones con un rezago de un año. La información se recolecta de "IMF International Finance Statistics".

Además, se utiliza el índice "Polity IV Project", para medir las características autoritarias de los países, como un proxy a los factores institucionales. La información se presenta en una escala de 21 puntos, que abarca desde -10 (monarquía hereditaria) hasta +10 (democracia consolidada) (Marshall, Monty y Jaggers, 2013).

Vale destacar que en la construcción de cualquier promedio ponderado, los pesos para cada país se crean a partir de su PIB en dólares internacionales.

Las principales características descriptivas de la muestra y las variables de interés se presentan en el cuadro 2.

CUADRO 2

ESTADÍSTICAS DESCRIPTIVAS (MEDIAS DE LA MUESTRA)

\begin{tabular}{ccc}
\hline & $\begin{array}{c}2 \text { puntos porcentuales del } \\
\text { PIB }\end{array}$ & $\begin{array}{c}5 \text { puntos porcentuales del } \\
\text { PIB }\end{array}$ \\
\hline Balance Cuenta Corriente/PIB & $-2,78 \%$ & $-2,70 \%$ \\
Crecimiento real PIB a 5 años & 3,63 puntos porcentuales & 5,40 puntos porcentuales \\
Crecimiento PTF a 5 años & 0,34 puntos porcentuales & 1,36 puntos porcentuales \\
Tasa de dependencia & 0,10 & 0,082 \\
Índice Chinn-Ito & 0,20 & 0,06 \\
Acuerdos Comerciales & $8,51 \%$ & $6,67 \%$ \\
Factores Institucionales & 3,25 & 2,27 \\
Reservas Internacionales/PIB & $14,24 \%$ & $17,06 \%$ \\
\hline
\end{tabular}

Fuente: Elaboración propia. 


\section{Modelos de supervivencia}

La metodología de análisis de supervivencia es rara vez empleada en el análisis de correcciones súbitas de cuenta corriente, por lo cual en esta sección se presenta un breve resumen de la metodología y algunas palabras clave. Esto con el fin de que el lector pueda comprender mejor los resultados obtenidos ${ }^{9}$.

Los modelos de supervivencia ${ }^{10}$ son una técnica inferencial que tiene como objetivo esencial modelar el tiempo que se tarda en que ocurra un determinado evento y estudia los factores que influyen en la duración de ocurrencia del evento. Es decir, estos modelos estudian los factores que afectan la extensión del tiempo que transcurre durante la transición de un estado a otro. El término "estado" se refiere a la clasificación de un agente en un momento del tiempo, "transición" es el movimiento de un estado a otro, $y$ una "duración" es el tiempo que se permanece en un determinado estado. Para el presente estudio, el evento es una reversión súbita del déficit de cuenta corriente, mientras la duración es el periodo anterior a este evento en el cual el país ha mantenido una secuencia de déficits de cuenta corriente.

Dentro de los modelos de supervivencia se conoce como la "tasa de riesgo", a la variable que indica la probabilidad de que cese un determinado estado dado que se ha sobrevivido hasta $t$. De igual manera, se entiende por "dependencia de la duración" al efecto que tiene el tiempo transcurrido desde el último evento, sobre la probabilidad de un nuevo evento. Así, una función de riesgo con pendiente positiva indica que la probabilidad de fallo en el momento $t$, condicional a la duración hasta el momento $t$ es creciente en $t$. Lo contrario ocurre con una pendiente negativa para la función de riesgo (Greene, 2012, p. 905).

Debido a la naturaleza del evento, existen consideraciones particulares a esta metodología. Estas consideraciones son: censura a la izquierda, censura a la derecha y regresores que varían con el tiempo. En general, la censura surge debido a que algunas transiciones se observan de manera incompleta. La censura a la derecha ocurre cuando se observa la duración desde el inicio del evento hasta un momento $c$, por lo tanto, solo se sabe que el final de la duración se encuentra en el intervalo $(c, \infty)$. En la base de datos utilizada en este estudio se presenta este tipo de censura, debido a que se debe imponer un año final (2012) pero para muchos países su déficit de cuenta corriente continúa sin enfrentar reversión. Por otro lado, la censura a la izquierda existe cuando un agente ha estado en el evento por un intervalo desconocido antes de ser observado. En nuestro caso, los países que antes de 1981 estaban en déficit de cuenta corriente son observaciones con censura a la izquierda, debido a que resulta muy difícil conocer el inicio de la duración.

La figura 1 puede ser ilustrativa para entender el concepto de censura. Pues ejemplifica las duraciones para Costa Rica presentes en la muestra. La duración 1 ya había iniciado antes de 1981, y puede resultar costoso determinar cuándo inició exactamente. Por lo tanto, esta duración presenta censura a la izquierda. Luego, la duración 2 comienza en 1985 y termina en 1991, mientras que la duración 3 inicia en 1993 y culmina en 2009. Ambas duraciones representan observaciones completas, por lo cual no se tiene ningún tipo de censura. Finalmente, en la duración 4 solo se conoce que el evento inicia en 2010, sin embargo no se cuenta con información de cuándo finalizará, por ende esta duración es un ejemplo de censura a la derecha.

9 Los modelos de supervivencia son un tema amplio, por lo cual se sugiere la lectura de Green (2012), en el cual se desarrolla con más detalle los modelos de supervivencia.

10 También llamados modelos de duración o análisis de sobrevivencia. 
FIGURA 1

CENSURA DE DATOS DE DURACIÓN (DATOS REALES PARA COSTA RICA)

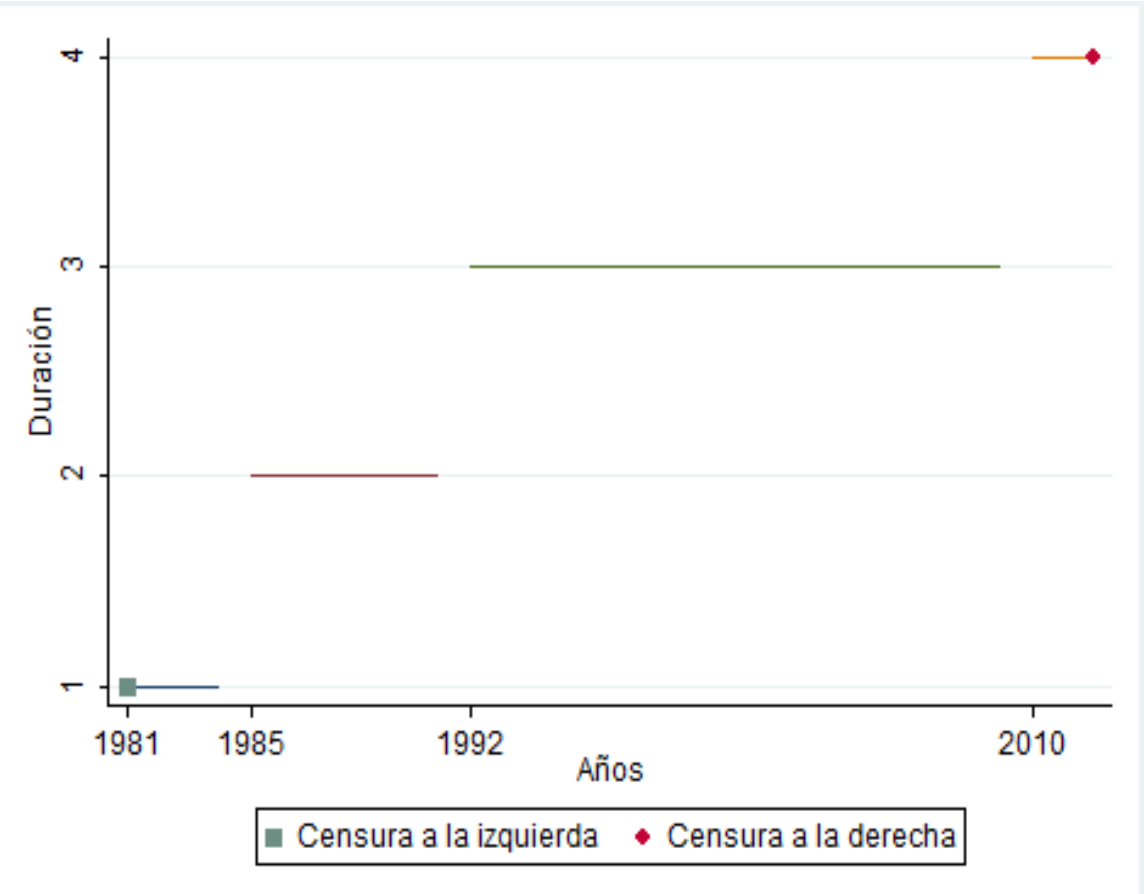

Fuente: Elaboración propia.

Econométricamente no existe un consenso para tratar la censura a la izquierda. Iceland (1997) presenta algunos métodos y hace hincapié en que el método "correcto" depende de cada investigación. Una opción es descartar la información que presenta dicha censura. Sin embargo, esto puede inducir a sesgos de selección en la muestra, además de que es ineficiente pues no se utiliza toda la información disponible. Otro método es estimar la duración con modelación estadística, pero usualmente implica partir de que la tasa de riesgo es constante. Esto puede ser un supuesto poco realista si el fenómeno se caracteriza por una tasa de riesgo que cambia a lo largo de la duración. Otra forma es comparar un modelo que incluya las observaciones con censura a la izquierda con otro que no, $y$ analizar las diferencias; sin embargo, este método en realidad no resuelve el problema de la censura. Finalmente, se podría redefinir la pregunta de investigación para evitar la presencia de censura a la izquierda, pero esto no siempre es posible.

Por el contrario, el problema de censura a la derecha es considerado menos serio. Allison (2010) explica que el método estándar para tratar censura a la derecha, es hacer el supuesto que dicha censura es "no informativa". Este supuesto significa que el hecho de que exista censura a la derecha no revela información valiosa sobre el riesgo de cambio de estado.

Finalmente, la particularidad de la metodología son los regresores que varían en el tiempo. Esto sucede debido a que como las unidades son observadas a lo largo del tiempo, algunos valores de las variables explicativas pueden cambiar. En el caso del presente estudio, variables como PIB real, crecimiento de la población, controles de capital, o cambios en reservas toman diferentes valores a lo 
largo del evento. Tratar esas variables como constantes es un problema de especificación dado que la historia de las variables explicativas puede ser relevante al explicar el proceso. Otra forma de abordar el problema es remplazar las variables con sus promedios a lo largo del evento. Asimismo, se puede dividir las observaciones totales en diferentes segmentos de acuerdo con las valores de las variables.

La regresión paramétrica en los modelos de supervivencia puede ser llevada a cabo utilizando distribuciones que incluyen la exponencial, Weibull, Gompertz, Log-normal, Log-logística, o Gamma. Como es señalado por Cameron y Trivedi (2009), el principal tema en la estimación paramétrica es la elección de la correcta especificación del modelo para obtener parámetros consistentes. Las estimaciones presentadas en este trabajo se basan en una distribución Weibull, sin embargo los resultados cambian muy poco al utilizar un Modelo Cox de Riesgo Proporcional (semi-paramétrico) El método de estimación para la distribución Weibull se basa en máxima verosimilitud, donde la función de verosimilitud es:

$$
\operatorname{In} L=\sum_{i}\left[\delta_{i}\left\{x_{i}^{\prime} \beta+\operatorname{In} \alpha+(\alpha-1) \operatorname{In} t_{i}-\exp \left(x_{i}^{\prime} \beta\right) t_{i}^{\alpha}\right\}-\left(1-\delta_{i}\right) \exp \left(x_{i}^{\prime} \beta\right) t_{i}^{\alpha}\right]
$$

Siendo si un indicador de censura a la derecha, tal que:

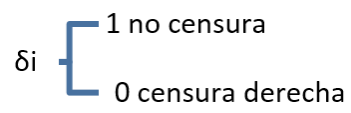

Y donde $x_{i}$ es un vector que incluye las variables explicativas, $\beta$ sus coeficientes asociados, $\alpha$ la dependencia de la duración y $t_{i}$ la duración.

Las condiciones de primer orden para $\alpha$ y $\beta$ son:

$$
\begin{gathered}
\frac{\partial \operatorname{In} L}{\partial \beta}=\sum_{i}\left[\delta_{i}-\exp \left(x_{i}^{\prime} \beta\right) t_{i}^{\alpha}\right] x_{i}=0 \\
\frac{\partial \operatorname{In} L}{\partial \alpha}=\sum_{i} \delta_{i}\left(\frac{1}{\alpha}+\operatorname{In} t_{i}\right)-\ln t_{i} \exp \left(x_{i}^{\prime} \beta\right) t_{i}^{\alpha}=0
\end{gathered}
$$

Es importante considerar que los modelos paramétricos se basan en distribuciones continuas, por ende el desarrollo anterior aplica para duraciones continuas (Cameron y Trivedi, 2009).

Un concepto clave en los modelos de supervivencia es la función de riesgo, la cual es la probabilidad de abandonar un estado condicional a sobrevivir al momento t. En el caso de una distribución Weibull la función de riesgo es $\gamma \alpha t^{\alpha-1}$ que es monótonamente creciente si $\alpha>1$ y monótonamente decreciente si $\alpha<1$ (cuando $\alpha=1$ la distribución Weibull es una exponencial) Otro concepto básico es la función de supervivencia: la probabilidad de que la duración iguale o exceda $t$. Una distribución Weibull tiene una función de supervivencia igual a $\exp \left(-\gamma t_{i}^{\alpha}\right)$.

Los resultados obtenidos en el documento también se mantienen al utilizar un Modelo Cox de Riesgos Proporcionales. Este es un método semi-paramétrico que no realiza ningún supuesto acerca de la distribución del tiempo de supervivencia. La especificación básica del modelo Cox de riesgos proporcionales:

$$
\mathrm{h}(\mathrm{t})=h_{0}(t) \ln t_{i} \exp \left(x^{\prime} \beta\right)=0
$$


donde $h_{0}(t)$ el término representa una función de riesgo de referencia arbitraria y no especificada para el tiempo continuo $t$ (Liu. 2012). La manera de encontrar los parámetros $\beta$ sin ningún supuesto sobre la forma funcional de la distribución es el uso de estimación por verosimilitud parcial.

Sin embargo, para ciertos fenómenos, la duración puede ser de naturaleza discreta o la forma en que se registra la información no permite conocer el momento exacto en que se presenta la transición (Jenkins, 2004). En estos casos se recurre a modelos de sobrevivencia discretos. Si la información sobre la duración es discreta por la forma en que se registran los datos (que es el caso de interés para el presente documento), la función de máxima verosimilitud viene dada por:

$$
L=\prod_{i}^{n} \prod_{j}^{j_{i}} h_{i j}^{y_{i j}}\left(1-h_{i j}\right)^{y_{i j}}
$$

donde es una variable que indica si el agente $i$ experimentó el evento en el momento $j$, con 1 si se experimentó y 0 en caso contrario, $y$ representa la probabilidad de que el agente $i$ enfrente el evento en el momento $j$, que viene dada por:

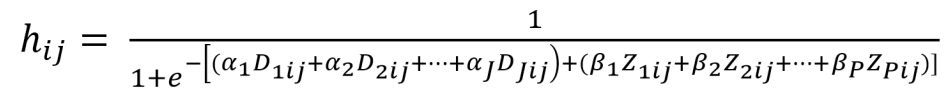

Las variables predictivas del modelo corresponden a $Z_{K i j}$, donde $\mathrm{K}=1, \ldots, P$ son $\mathrm{P}$ predictores, del agente $i$ en el momento $j$. Mientras que $D_{s i j}$ con $s=1, \ldots, J$, es una sucesión de variables dicotómicas, que indican periodos de tiempo. De esta forma, $=1$ si $s=j$ y 0 en otro caso. Por último, $\alpha_{s}$ y $\beta_{K}$ son parámetros asociados a dichas variables.

Singer y Willet (1993) mencionan trabajos donde se demuestra que existe una equivalencia entre la función de verosimilitud para sobrevivencia en variable discreta y el modelo de pruebas independientes de Bernoulli, lo cual permite utilizar los resultados de regresión logística (Singer \& Willet, 1993). Esta es la razón por la que el análisis de supervivencia de datos discretos se puede estimar mediante modelos de decisión binaria, como probit, logit, o cloglog. Entre los tres modelos, la decisión de cuál elegir suele considerarse de poca importancia, pues sus resultados se consideran similares (Golder, 2013).

Para la estimación de un modelo de supervivencia de variable discreta se crea una variable que indica si el evento ha finalizado o se encuentra en proceso, y que sirve como variable dependiente en la estimación del modelo de decisión binaria. El valor de 1 indica que la observación está censurada y 0 que no lo está.

Además, es necesario especificar variables que aproximen la dependencia de la duración, es decir cómo se afecta la probabilidad de fallo por el transcurrir del tiempo. Es importante notar que los modelos de decisión binaria no consideran por sí solos los efectos de la dependencia de la duración. El no tener en cuenta este factor puede llevar a estimaciones erróneas. Para incorporar la dependencia de la duración en los modelos de decisión binaria se pueden utilizar los siguientes métodos (Golder, 2013):

1. Corregir los errores estándar: por ejemplo, mediante el uso de errores estándar robustos.

2. Incluir variables dicotómicas de tiempo: se trata de utilizar variables dicotómicas que indiquen cada duración. No obstante, tiene el problema de que la inclusión de variables dicotómicas puede restarle grados de libertad a la estimación.

3. Realizar transformaciones en la duración: consiste en incluir como variables explicativas transformaciones de la duración de los eventos. Un problema potencial es especificar una relación funcional para la dependencia de la duración errónea. Una dependencia de la duración similar a la que se sigue en una distribución Weibull continua, se consigue con el logaritmo 
de la duración. Por otra parte, no es necesario incluir una variable de duración si se considera que la dependencia de la duración es constante (StataCorp, 2013).

Tanto en el modelo de variable continua como discreta, es importante considerar la heterogeneidad no observable, es decir, la posibilidad de que existan diferencias entre los individuos que no estén recogidas en los datos disponibles. Dicho problema puede ocurrir por razones como variables omitidas o problemas de medición (Jenkins, 2004). Es por ello necesario realizar una modificación a los modelos desarrollados anteriormente para incorporar la heterogeneidad no observable. Una forma de incorporar esa heterogeneidad no observada en el caso de modelos con duración continua es suponiendo una determinada distribución para dichas variables no observadas u omitidas. Esta incorporación conlleva a una generalización de los modelos de supervivencia (Gutiérrez, 2002). En el caso discreto se recurre a modelos de efectos aleatorios.

\section{Aplicación}

Se define una reversión de cuenta corriente en el momento $t$ como una reducción del déficit de cuenta corriente de al menos 2 puntos porcentuales del PIB; para efectos comparativos, se realiza el análisis considerando además una reducción de 5 puntos porcentuales del PIB. El momento en que termina la duración es cuando el país se encuentra en déficit y experimenta una reversión repentina, es decir de un año a otro.

La forma en que se trató el problema de la censura a la izquierda en este trabajo fue descartar dichas observaciones. Como se mencionó antes, pueden surgir algunas limitaciones por sesgos de selección. Respecto a la censura a la derecha, se utiliza el supuesto de que la censura es no informativa.

Los regresores que cambian con el tiempo incluyen variables son PIB, tasa de dependencia, controles de capital o cambios en reservas. El considerar dichas variables como constantes puede llevar a problemas de especificación. En el presente documento las observaciones correspondientes a dichas variables se dividen en varios segmentos. Por ejemplo, considere el caso de la tasa de dependencia en Costa Rica, un evento inicia en 1985 y finaliza en 1991, entonces la información se registra como:

CUADRO 1

PROCEDIMIENTO PARA TRATAR CON VARIABLES QUE CAMBIAN EN EL TIEMPO

\begin{tabular}{ccc}
\hline Año & Tasa de Dependencia & $\begin{array}{c}\text { Indicador de Censura } \\
(1=\text { censurada })\end{array}$ \\
\hline 1985 & 0,755 & 0 \\
1986 & 0,754 & 0 \\
1987 & 0,754 & 0 \\
1988 & 0,756 & 0 \\
1989 & 0,759 & 0 \\
1990 & 0,764 & 0 \\
1991 & 0,771 & 1 \\
\hline
\end{tabular}

Fuente: Elaboración propia. 
El evento finaliza en 1991, entonces la información se registra como si seis observaciones fueran censuradas y la sétima completa.

Para las estimaciones se utiliza una distribución Weibull, que fue introducida antes. Los resultados no cambian si se utiliza un Modelo Cox de Riesgo Proporcional. Además, se considera la pertinencia de efectos no observados en los datos. Entre las maneras de introducir dichos efectos puede ser suponer una distribución gamma o una Gausiana Inversa. Con cualquier distribución los resultados se mantienen, pero por criterio de Log verosimilitud se decide presentar los derivados de la distribución gamma.

La forma de incluir la dependencia de la duración en la estimación de variable discreta es mediante el logaritmo de la duración. Se decide utilizar este método, ya que según las estimaciones con distribución Weibull la dependencia de la duración es un aspecto importante en la determinación de la probabilidad de reversión. El incluir el logaritmo de la duración es la forma de obtener un patrón similar en un modelo discreto, como se comentó anteriormente.

\section{RESULTADOS}

La primera estimación se basa en una reversión de cuenta corriente de al menos 2 puntos porcentuales del PIB. Se cuentan con 2581 observaciones y 18\% de ellas ha sido una reversión. Los resultados de la estimación se muestran en el cuadro 3.

La primera variable que muestra tener relevancia para la duración del déficit de cuenta corriente es el crecimiento del PIB real futuro a 5 años. A mayor crecimiento en relación al mundo, la probabilidad de una reversión se reduce. Este resultado es consistente con el enfoque intertemporal de la cuenta corriente. De acuerdo con esta teoría, un incremento en el ingreso futuro reduciría los ahorros presentes, entonces el déficit de la cuenta corriente debería tener una mayor extensión temporal. La figura 2 presenta la función de supervivencia para un crecimiento del PIB real futuro a 5 años de $-2,05$ puntos porcentuales y de 15,68 puntos porcentuales. Es claro observar que conforme el crecimiento futuro sea mayor, la probabilidad de que no se enfrente una reversión al transcurrir el tiempo se incrementa.

FIGURA 2

FUNCIÓN DE SOBREVIVENCIA PARA EL CRECIMIENTO DEL PIB REAL A 5 AÑOS

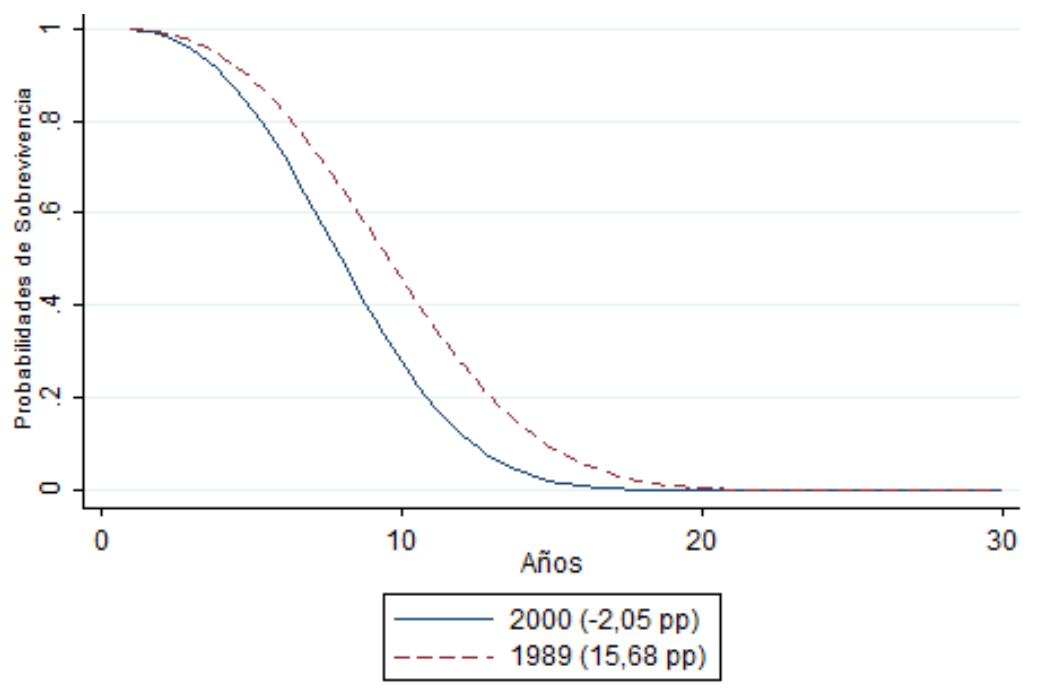

Fuente: Elaboración propia. 
Por otra parte, el crecimiento de la PTF tiene un efecto directo en la probabilidad de reversiones de cuenta corriente. En otras palabras, el incremento en la PTF aumenta la probabilidad de reversión. Este resultado puede ser un ejemplo de la paradoja de Feldstein-Horioka. Esta paradoja es el descubrimiento hecho en 1980 por dichos autores, y se refiere a que entre países de la OCDE la tasa de ahorro nacional está altamente correlacionada con la tasa de ahorro doméstico. Además, regresiones de la inversión en los ahorros evidenciaron coeficientes cercanos a la unidad. Esto ha originado múltiples artículos tratando de ofrecer una respuesta a lo que originaba dichos resultados. De hecho, esta paradoja fue catalogada por Obsfeld $y$ Rogoff como uno de los mayores acertijos en macroeconomía internacional (Obstfeld \& Rogoff, 2000).

\section{CUADRO 3 \\ ESTIMACIÓN}

\begin{tabular}{|c|c|c|}
\hline Variable & $\begin{array}{l}\text { Reversión de al menos } 2 \text { puntos } \\
\text { porcentuales del PIB }\end{array}$ & $\begin{array}{c}\text { Reversión de al menos } 5 \text { puntos por- } \\
\text { centuales del PIB }\end{array}$ \\
\hline \multirow[t]{2}{*}{ Crecimiento real PIB a 5 años $(*)$} & $-0,028 * * *$ & $-0,050$ *** \\
\hline & $(0,005)$ & $(0,010)$ \\
\hline \multirow[t]{2}{*}{ Crecimiento PTF a 5 años $\left({ }^{*}\right)$} & $0,027 * * *$ & $0,064 * * *$ \\
\hline & $(0,005)$ & $(0,011)$ \\
\hline \multirow[t]{2}{*}{ Tasa de dependencia } & $-4,758 * *$ & $-2,082$ \\
\hline & $(2,107)$ & $(4,054)$ \\
\hline \multirow[t]{2}{*}{ Índice de Control de Capitales (*) } & $-0,010$ & 0,061 \\
\hline & $(0,057)$ & $(0,094)$ \\
\hline \multirow[t]{2}{*}{ Cuenta Corriente Regional (*) } & $-0,014$ & $-0,048 * *$ \\
\hline & $(0,013)$ & $(0,022)$ \\
\hline \multirow[t]{2}{*}{ Acuerdos Comerciales $(*)$} & $-0,057 * * *$ & $-0,041 *$ \\
\hline & $(0,009)$ & $(0,015)$ \\
\hline \multirow[t]{2}{*}{ Factores Institucionales } & $-0,032 * *$ & $-0,107 * * *$ \\
\hline & $(0,013)$ & $(0,023)$ \\
\hline \multirow[t]{2}{*}{ Cambios en Reservas $(*)$} & $-0,004 * *$ & $-0,001$ \\
\hline & $(0,001)$ & $(0,001)$ \\
\hline \multirow[t]{2}{*}{ Balance de cuenta corriente en el periodo anterior } & $-0,071^{* * * *}$ & $-0,103 * * *$ \\
\hline & $(0,006)$ & $(0,010)$ \\
\hline \multirow[t]{2}{*}{ Constante } & $-5,269 * * *$ & $-6,814 * * *$ \\
\hline & $(0,266)$ & $(0,450)$ \\
\hline \multirow[t]{2}{*}{ Dependencia de la duración (Logaritmo) } & $1,016^{* * *}$ & $0,886^{* * *}$ \\
\hline & $(0,034)$ & $(0,058)$ \\
\hline \multirow[t]{2}{*}{ Varianza de los efectos no observados } & $1,072 * * *$ & $1,499 * * *$ \\
\hline & $(0,163)$ & $(0,304)$ \\
\hline $\mathrm{N}$ & 2581 & 1713 \\
\hline Log-likelihood & $-837,334$ & $-379,832$ \\
\hline Wald chi2(9) & 308,576 & 237,92 \\
\hline \multicolumn{3}{|l|}{$\begin{array}{l}\text { Desviación estándar en paréntesis } \\
(*) \text { Variable rezagada un año }\end{array}$} \\
\hline \multicolumn{3}{|c|}{ Niveles de Significancia: *** $1 \% * * 5 \% * 10 \%$} \\
\hline
\end{tabular}


En relación al presente estudio, si los incrementos en la PTF están relacionados con mayores niveles de inversión, de acuerdo con la paradoja los ahorros nacionales deberían incrementarse también. Por lo tanto, el déficit de cuenta corriente debe disminuir. La figura 1 del anexo 2 presenta un gráfico de la PTF para un crecimiento de -8,52 puntos porcentuales y 8,91 puntos porcentuales respecto al cambio en la productividad mundial. Muestra que un país con un mayor crecimiento de la PTF es menos probable que continúe sin una reversión en el déficit de cuenta corriente que un país con un menor crecimiento de la PTF futura.

Otra posible explicación para el signo positivo del coeficiente de la PTF es por vía del tipo de cambio real, mediante lo que en la literatura se conoce como el efecto Harrod-Balassa-Samuelson (HBS), según el cual un aumento de la productividad está asociado con apreciaciones reales, las cuales pueden llegar a reducir o eliminar los déficit de cuenta corriente ${ }^{11}$.

Un factor demográfico que ha evidenciado tener un efecto significativo en la duración del déficit de cuenta corriente es la tasa de dependencia. Teóricamente, la hipótesis del ciclo de vida argumenta que el crecimiento en la población inactiva causaría una reducción en los ahorros, puesto que la economía tendría un incremento en la cantidad de personas que consumen más de lo que producen (Rose, Supaat \& Braude, 2009). Por lo tanto, de acuerdo con esta hipótesis, se podría esperar un incremento en el déficit de cuenta corriente junto a un envejecimiento de la población. El efecto se encuentra en ambas estimaciones, pero solo para la reversión de 2 puntos porcentuales es estadísticamente significativo.

La variable acuerdos comerciales, que representa el porcentaje del PIB mundial con el cual se han ratificado acuerdos comerciales, sugiere un impacto positivo, es decir, reduce la probabilidad de una reversión súbita en la cuenta corriente. Milesi-Ferrett y Razin (1997) utilizaron la variable apertura comercial y encontraron un efecto similar en la probabilidad de reversión de cuenta corriente, en un estudio realizado para países de ingreso bajo y medio. Ellos explican que una economía abierta tendría menos dificultades en afrontar deudas con el exterior y menos incentivos para no pagar los débitos externos. Así, la misma lógica se puede aplicar a nuestros resultados, dado que un acuerdo comercial refuerza el compromiso de un país con la apertura comercial. La figura 3 en el Anexo 2 muestra que un país con acuerdos comerciales con un grupo de países que represente cerca de un $26 \%$ del PIB mundial tiene mayores probabilidades de no enfrentar una reversión de cuenta corriente que un país que no posea acuerdos comerciales.

La variable cuenta corriente regional trata de capturar el impacto de la cuenta corriente de países vecinos. Las estimaciones muestran que existe una relación inversa entre la cuenta corriente regional y las probabilidades de reversión. Por ejemplo, si el déficit de la cuenta corriente regional se incrementa, la probabilidad de reversión también crece. Una posibilidad es que los países sean considerados como sustitutos cercanos entre inversionistas y, en consecuencia, si algún país en el bloque está en problemas para pagar sus deudas con el exterior, también se consideran casos peligrosos. Kintanar (2009) encuentra significativa una medida de sensibilidad de un país ante shocks en la cuenta corriente de sus vecinos, $y$ con un efecto en el mismo sentido. Como se muestra en la figura 4, en el Anexo 2, es más probable que un país no enfrente una reversión cuando los países considerados similares tienen una cuenta corriente superavitaria. Vale señalar que para el caso de las estimaciones de al menos 2 puntos porcentuales del PIB, el efecto no es estadísticamente significativo.

11 El signo positivo del coeficiente asociado a la PTF futura podría ser explicado por otros factores tales como: (i) los mercados de capitales imperfectos o un riesgo país alto, pues hacen que la economía interna deba ahorrar más para financiar el nuevo requerimiento de inversión, reduciendo de esta forma el déficit de cuenta corriente; (ii) una relación de causalidad inversa, es decir, que los incrementos en la PTF no incrementen la probabilidad de corrección, sino que una corrección de cuenta corriente hoy, genere los incentivos para que los insumos productivos (trabajo y capital) sean reasignados entre sectores, lo cual incrementaría la productividad de la economía en el futuro. 
Finalmente, a mayores cambios en reservas, las probabilidades de reversión de cuenta corriente son menores. Este hallazgo es coherente con la mayoría de estudios presentados en la sección 2. Cuando un país enfrenta una reducción en su nivel de reservas internacionales con relación al cambio del resto del mundo, puede generar una pérdida de confianza y hacer más probable una reversión, como se constata en la figura 5.

El índice de control de capitales parece tener un efecto ambiguo en la probabilidad de reversiones. Por una parte, para la estimación que involucra 2 puntos porcentuales del PIB, la reducción de controles de capital sugiere reducir las probabilidades de reversión. Sin embargo, para las estimaciones de reversiones de al menos 5 puntos porcentuales de PIB, el efecto de la variable sugiere lo contrario. Vale señalar que para ambas estimaciones el efecto no posee significancia estadística. Estos resultados dan argumentos para críticos y promotores de la liberalización financiera. Edwards (2005) estudió la misma relación, pero no encontró evidencia de que países con mayor movilidad de capital tuvieran una menor incidencia de crisis respecto a países con menor movilidad. Sin embargo, encontró que una vez que una crisis sucede, los países con mayor movilidad de capital enfrentan un mayor costo en términos de reducción de producto. Mediante una estimación probit, Eichengreen y Adalet (2005) encontraron que la incidencia de controles de capital limita las reversiones de cuenta corriente. No obstante, la variable no es estadísticamente significativa. El Trade and development report, 2008 presenta como los resultados de una estimación logit que una cuenta de capitales abierta reduce las probabilidades de una reversión de cuenta corriente, siendo estadísticamente significativo su efecto (United Nations Conference on Trade and Development, 2008). La figura 6 presenta el caso para un control de capitales de -1,86 y de 2,44, en la estimación de al menos 2 puntos porcentuales. Es más probable que un país continúe sin una reversión de cuenta corriente cuando los controles de capital se debilitan.

Un resultado importante, estadísticamente significativo en ambas estimaciones, es que la dependencia de la duración es mayor que uno (logaritmo mayor que cero). Ello sugiere que la dependencia de la duración es positiva, es decir, si un país se encuentra en déficit de cuenta corriente, la probabilidad de que se enfrente a una reversión se incrementa conforme transcurre el tiempo. Asimismo, la varianza de los efectos aleatorios es estadísticamente distinta de cero en ambas estimaciones, lo que sugiere la pertinencia de corregir por heterogeneidad no observable. Vale señalar que debido al criterio de Log verosimilitud se decide corregir heterogeneidad no observable mediante una distribución Gamma.

Utilizando las estimaciones es posible obtener la probabilidad de reversión de cuenta corriente para Costa Rica. Se inicia con las predicciones dentro de muestra, los resultados se presentan en el cuadro 4. 
CUADRO 4

PROBABILIDADES DE REVERSIÓN DE CUENTA CORRIENTE, ESTIMACIONES DENTRO DE MUESTRA $(1985-2012)$

\begin{tabular}{|c|c|c|c|c|}
\hline Año & $\begin{array}{l}\text { Probabilidad de re- } \\
\text { versión de al menos } 2 \\
\text { p.p. del PIB ( } \%)\end{array}$ & $\begin{array}{l}\text { Probabilidad de re- } \\
\text { versión de al menos } 5 \\
\text { p.p. del PIB (\%) }\end{array}$ & Reversión & DCC/PIB (\%) \\
\hline 1985 & 0,76 & 0,4 & No & $-3,31$ \\
\hline 1986 & 2,49 & 0,59 & No & $-1,68$ \\
\hline 1987 & 5,03 & 0,65 & No & $-5,05$ \\
\hline 1988 & 11,32 & 1,36 & No & $-3,47$ \\
\hline 1989 & 10,98 & 1,32 & No & $-7,20$ \\
\hline 1990 & 22,4 & 2,3 & No & $-7,83$ \\
\hline 1991 & 33,52 & 2,71 & Sí & $-3,13$ \\
\hline 1992 & 0,59 & 2,08 & No & $-4,77$ \\
\hline 1993 & 2,45 & 2,31 & No & $-7,16$ \\
\hline 1994 & 6,21 & 3,11 & Sí & $-4,86$ \\
\hline 1995 & 0,87 & 3,43 & No & $-3,18$ \\
\hline 1996 & 2,02 & 2,75 & No & $-2,32$ \\
\hline 1997 & 3,3 & 2,04 & No & $-3,59$ \\
\hline 1998 & 5,82 & 3,24 & No & $-3,48$ \\
\hline 1999 & 11,12 & 4,38 & No & $-3,85$ \\
\hline 2000 & 13,12 & 4,78 & No & $-4,50$ \\
\hline 2001 & 19,26 & 4,82 & No & $-3,68$ \\
\hline 2002 & 23,35 & 5,77 & No & $-5,09$ \\
\hline 2003 & 28,58 & 6,28 & No & $-5,02$ \\
\hline 2004 & 28,35 & 5,06 & No & $-4,25$ \\
\hline 2005 & 33,59 & 5,25 & No & $-4,91$ \\
\hline 2006 & 37,63 & 6,16 & No & $-4,54$ \\
\hline 2007 & 41,77 & 6,24 & No & $-6,25$ \\
\hline 2008 & 16,58 & 3,21 & No & $-9,34$ \\
\hline 2009 & 29,06 & 6,01 & Sí & $-1,96$ \\
\hline 2010 & 0,16 & 0,03 & No & $-3,53$ \\
\hline 2011 & 0,57 & 0,1 & No & $-5,37$ \\
\hline 2012 & 1,38 & 0,26 & No & $-5,27$ \\
\hline
\end{tabular}

Fuente: Elaboración propia. 
Los resultados presentados en el cuadro 4, deben ser entendidos como la probabilidad de que se dé una corrección abrupta del déficit de cuenta corriente para ese año en particular, con base en los modelos estimados. El incremento o disminución de la probabilidad, indica que una corrección es más o menos probable, sin embargo al ser una variable estocástica, su interpretación debe ser tomada con prudencia, pues pueden existir otros factores no incluidos en el modelo, que pueden tener efecto sobre la realización o no de la corrección.

En este sentido las proyecciones de estos modelos deben ser analizados de manera conservadora, tomando en cuenta una lectura de la condición de la economía como un todo. Esto con el fin de no caer en alarmas innecesarias o un nivel de complacencia excesiva. Sin embargo los resultados de los modelos enriquecen el análisis de política al proveer al hacedor de política con argumentos numéricos sobre el posible comportamiento de una variable tan importante como lo es la Cuenta Corriente.

Las estimaciones fuera de muestra se realizan para los años 2013-2017. Los supuestos que se siguen son:

1. El índice de control de capitales se mantiene constante en su nivel alcanzado en 2011, asumiendo un valor de 2,44. En el 2014, se aprobó un proyecto de ley en la Asamblea Legislativa denominado "Ley para desincentivar el ingreso de capitales externos". Este proyecto busca limitar el ingreso de capitales de corto plazo atraídos por diferenciales de tasas de interés que generen presiones al tipo de cambio, imponiendo medidas cuando las condiciones así lo ameriten. Con los nuevos controles de capital el índice podría disminuir en los años posteriores a 2011.

2. Los factores institucionales se mantienen constantes. En otras palabras, Costa Rica no cambia su estatus de democracia consolidada.

3. Las proyecciones del balance de cuenta corriente regional se realizan con las estimaciones del World Economic Outlook de octubre 2013.

4. Las estimaciones de PTF se toman del Econmap del CEPII.

5. El crecimiento del PIB real se toma como el promedio de los últimos 5 años.

6. Se supone, además, que durante el periodo 2014-2017 no se ratificarían nuevos acuerdos comerciales. Consideramos que este supuesto es razonable, ya que acuerdos como los tratados de libre comercio deben ser aprobados por la Asamblea Legislativa, y el tiempo promedio en Costa Rica para que una ley sea aprobada es de 35,7 meses (Programa Estado de la Nación, 2012).

El cuadro 6 presenta las probabilidades de reversión de cuenta corriente de acuerdo con los supuestos. Los resultados sugieren que Costa Rica no tiene una alta probabilidad de una reversión, al menos en los próximos cinco años. No obstante, la veracidad de los supuestos decae con el tiempo, así que las estimaciones más confiables son para los años 2013 y 2014. 
CUADRO 5

COSTA RICA: PROBABILIDADES DE REVERSIÓN DE CUENTA CORRIENTE, ESTIMACIONES FUERA DE MUESTRA (2013-2017)

\begin{tabular}{cccc}
\hline Año & $\begin{array}{c}\text { Probabilidad de reversión } \\
\text { de al menos 2 p.p. (\%) }\end{array}$ & $\begin{array}{c}\text { Probabilidad de reversión } \\
\text { de al menos 5 p.p. (\%) }\end{array}$ & DCC/PIB (\%) \\
\hline 2013 & 0,73 & 0,17 & $-5,53$ \\
2014 & 1,36 & 0,29 & $-5,47$ \\
2015 & 0,6 & 0,17 & $-5,64$ \\
2016 & 0,78 & 0,22 & $-5,61$ \\
2017 & 0,96 & 0,27 & $-5,70$ \\
\hline
\end{tabular}

Fuente: Elaboración propia.

La figura 3 presenta las probabilidades estimadas.

FIGURA 3

PROBABILIDAD DE REVERSIÓN DE CUENTA CORRIENTE

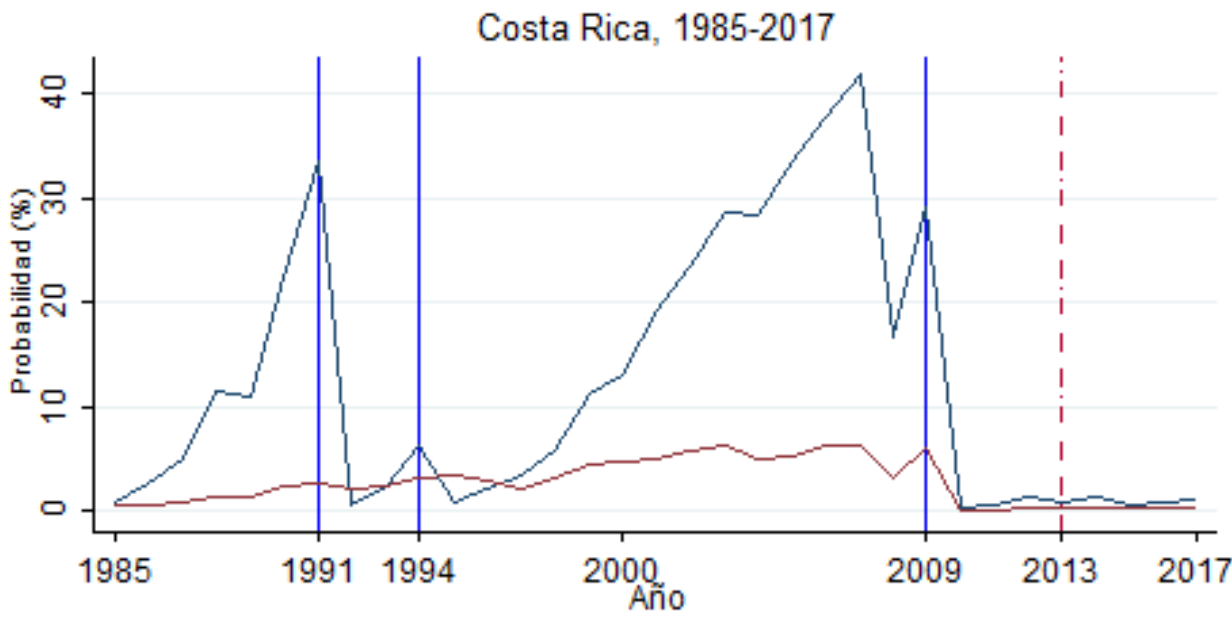

Reversión de 2 pts porcentuales del PIB Reversión de 5 pts porcentuales del PIB

La línea vertical azul indica una reversión de Cuenta Corriente

La línea vertical roja indica donde las proyecciones de datos inician

Fuente: Elaboración propia. 
Utilización de un modelo de variable discreta

La sección 6 basa las estimaciones y resultados en un modelo de variable continua. Sin embargo, a pesar de que el desarrollo de la cuenta corriente ocurre en un intervalo continuo de tiempo, sus registros en la base de datos están en intervalos de un año. Ello implica que se trabajan con variables discretas. Hess y Persson (2010) analizan estudios sobre la duración de flujos comerciales, y señalan que a pesar de que la mayoría de investigaciones se basan en el Modelo Cox de Riesgo Proporcional (de variable continua), econométricamente se puede estar cometiendo un error (Hess \& Persson, 2010). Para evaluar cómo cambian las conclusiones derivadas anteriormente, en esta sección se re-estima el modelo con métodos de supervivencia para variable discreta.

En la sección 3 se abordaron los aspectos metodológicos pertinentes. Además, para considerar el efecto de heterogeneidad no observable, se recurre a un modelo de efectos aleatorios. En estudios como Hess y Persson (2010), se concluyó que este tipo de modelos dan una mejor estimación que el Modelo Cox de Riesgo Proporcional para variable continua. De los modelos de decisión binaria estimados (probit, logit y complementaria log-log) debido al criterio de Log verosimilitud se decide realizar el análisis con el modelo probit.

El cuadro 6 presenta las estimaciones para el caso de reversiones de al menos 2 puntos porcentuales del PIB.

CUADRO 6

ESTIMACIÓN (VARIABLE DISCRETA)

\begin{tabular}{ccc}
\hline Variable & $\begin{array}{c}\text { Reversión de al menos 2 pun- } \\
\text { tos porcentuales del PIB }\end{array}$ & $\begin{array}{c}\text { Reversión de al menos 5 } \\
\text { puntos porcentuales del PIB }\end{array}$ \\
\hline Crecimiento real PIB a 5 años $\left(^{*}\right)$ & $-0,015^{* * *}$ & $-0,020 * * *$ \\
Crecimiento PTF a 5 años $(*)$ & $(0,004)$ & $(0,006)$ \\
Tasa de dependencia & $0,017 * * *$ & $0,028 * * *$ \\
& $(0,004)$ & $(0,007)$ \\
Índice de Control de Capitales $(*)$ & $-2,277^{*}$ & $-0,537$ \\
& $(1,060)$ & $(1,788)$ \\
Cuenta Corriente Regional $(*)$ & $-0,032$ & 0,069 \\
Acuerdos Comerciales $(*)$ & $(0,031)$ & $(0,043)$ \\
& $-0,004$ & $-0,018$ \\
Factores Institucionales & $(0,008)$ & $(0,012)$ \\
& $-0,016 * * *$ & $-0,007$ \\
Cambios en Reservas $(*)$ & $(0,005)$ & $(0,007)$ \\
& $-0,011$ & $-0,034 * * *$ \\
& $(0,008)$ & $(0,011)$ \\
& $-0,003 * * *$ & $-0,001$ \\
& $(0,001)$ & $(0,001)$
\end{tabular}


Balance de cuenta corriente en el periodo anterior

Logaritmo duración

Constante

$\mathrm{N}$

Log-likelihood

Wald chi2(9)
$-0,094 * * *$

$(0,006)$

$0,623 * * *$

$(0,061)$

$-1,826 * * *$

$(0,134)$

2581

$-933,50$

351,68
$-0,089 * * *$

$(0,008)$

0,267 ***

0,082

$-2,26^{* * *}$

$(0,223)$

1713

$-387,12$

157,53

Desviación estándar en paréntesis

(*) Variable rezagada un año

Niveles de Significancia: *** $1 \% * * 5 \% * 10 \%$

Fuente: Elaboración propia.

Los efectos de todas las variables se mantienen en el caso del probit con efectos aleatorios. Sin embargo, la variable factores institucionales deja de ser estadísticamente significativa.

Por otra parte, las estimaciones para el caso de reversión de al menos 5 puntos porcentuales del PIB, se presenta en el cuadro 8.

En comparación con la estimación de variable continua, la variable acuerdos comerciales y el balance de la cuenta corriente regional pierden significancia estadística. No obstante, los efectos mantienen su dirección.

El logaritmo de la duración, en ambas estimaciones sugiere que la probabilidad de reversión aumenta conforme pasa el tiempo, lo cual también se encontró al utilizar métodos para duraciones en variable continua.

Las probabilidades estimadas según ambos modelos tienden a ser similares, de hecho para la reversión de al menos 2 puntos porcentuales del PIB, el índice de correlación es 0,92; y para el caso de la reversión de al menos 5 puntos porcentuales del PIB, es de 0,81. La figura 4 muestra el comportamiento de las probabilidades estimadas con el modelo de variable discreta, mientras que la figura 5 presenta las 4 series de probabilidades estimadas. 
FIGURA 4

PROBABILIDAD DE REVERSIÓN DE CUENTA CORRIENTE

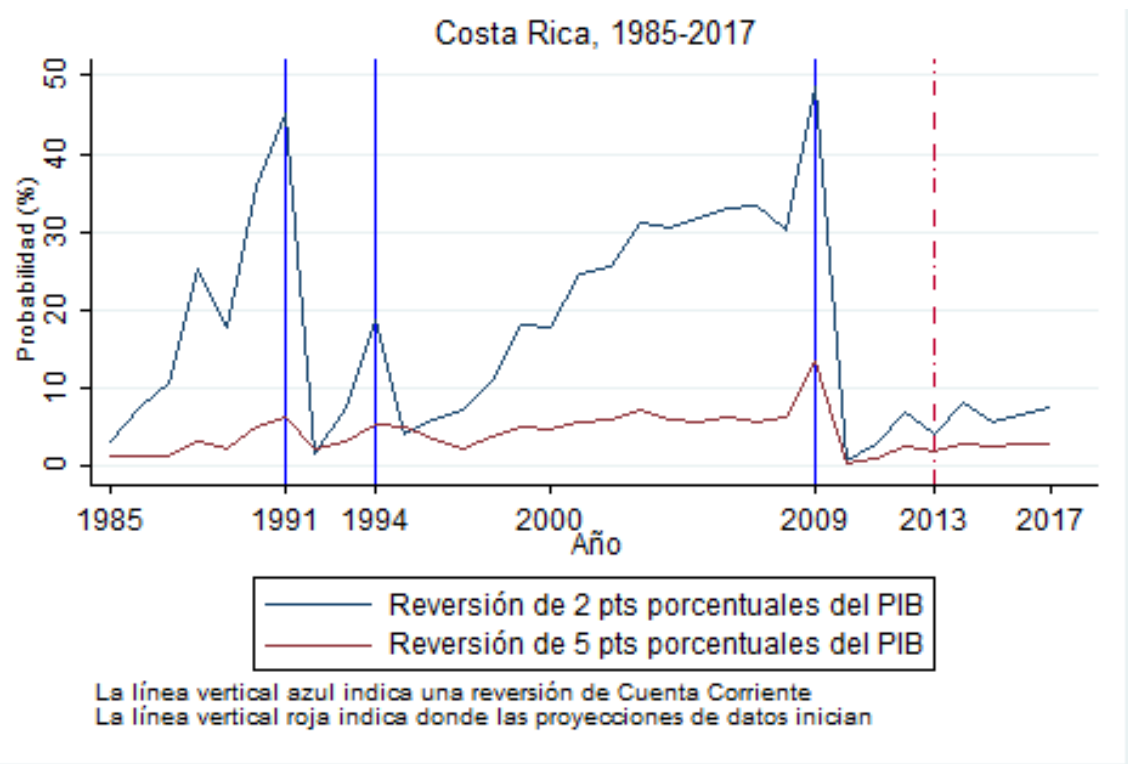

Fuente: Elaboración propia.

Para el periodo 2013-2017, la probabilidad de que la economía costarricense experimente una corrección súbita del déficit de cuenta corriente del orden de 2 p.p. del PIB es inferior al 10\%.

FIGURA 5

PROBABILIDAD DE REVERSIÓN DE CUENTA CORRIENTE
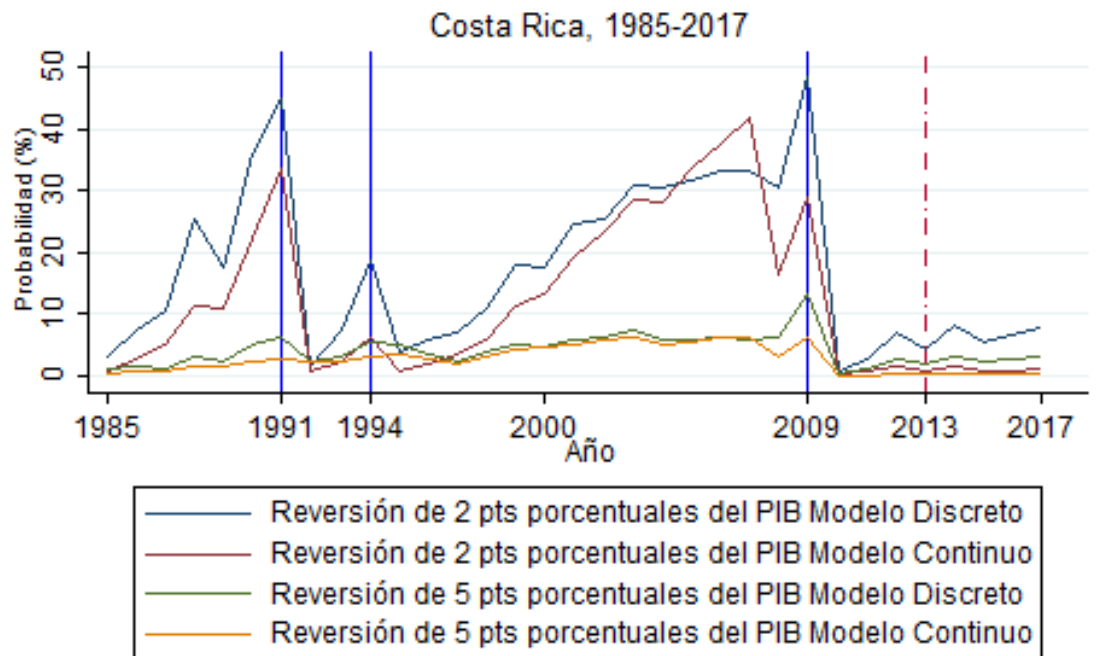

La línea vertical azul indica una reversión de Cuenta Corriente

La línea vertical roja indica donde las proyecciones de datos inician

Fuente: Elaboración propia. 
En la figura 5, se puede observar como ambas metodologías muestran un comportamiento similar, lo cual es esperable pues tanto el Modelo Discreto como el Continuo poseen variables explicativas similares y los coeficientes coinciden en su signo. Sin embargo, la interpretación no es idéntica, pues para el caso continuo existe una dependencia sobre el tiempo que la economía se ha mantenido sin correcciones de cuenta corriente.

A pesar de las diferencias observadas en la figura 5, ambas metodologías muestran resultados congruentes. Se considera que el análisis comparativo de los resultados de ambos modelos provee una interpretación más robusta del estado de la economía, que el empleo de solamente una de las metodologías. Esto por cuanto los modelos utilizan diversas metodologías, para las cuales no hay una prueba estadística que indique que uno u otro es preferible.

Al ser un análisis con datos de panel, el ajuste al caso particular de Costa Rica tiende a ser bajo. Sin embargo, se considera que las predicciones muestran un ajuste razonablemente bueno para ser un indicador de riesgos para la estabilidad de la cuenta corriente. Es importante destacar que debido a la gran cantidad de choques que pueden afectar el comercio internacional, existe un significativo grado de incertidumbre sobre el desempeño futuro de la cuenta corriente, por lo cual se estima que las proyecciones de estos modelos deben ser interpretadas de manera conservadora, por los hacedores de política.

\section{CONCLUSIONES}

El documento estimó la probabilidad de correcciones súbitas en el déficit de cuenta corriente, enfocándose especialmente en Costa Rica. Se hace uso de modelos de supervivencia, definiendo una reversión de cuenta corriente como una reducción en el déficit de cuenta corriente de al menos: 2 puntos porcentuales del PIB; alternativamente se evaluó una reducción de 5 puntos porcentuales del PIB.

Los resultados sugieren que las probabilidades de reversión de un déficit de cuenta corriente están afectadas de manera inversa por el crecimiento del PIB real a 5 años en relación al resto del mundo, de una mejora en el balance de cuenta corriente de países considerados similares, un incremento en la tasa de dependencia, un aumento en el porcentaje del PIB mundial con el que se tienen acuerdos comerciales, mayor acumulación de reservas y factores institucionales más democráticos.

Un incremento en el crecimiento de la productividad total de los factores (PTF) a 5 años incrementa la probabilidad de una reversión. Se tiene la hipótesis de que este resultado puede deberse a la paradoja Feldstein-Horioka; sin embargo, para poder concluir al respecto, debe realizarse un mayor análisis que escapa al objetivo de este estudio.

Finalmente, los controles de capital sugieren resultados ambiguos, aunque nunca estadísticamente significativos. Cuando la regresión se realiza para reversiones de al menos 2 puntos porcentuales del PIB, la reducción en los controles de capital sugieren disminuir la probabilidad de reversiones. Sin embargo, la regresión que estima reversiones de al menos 5 puntos porcentuales del PIB muestra que una reducción en los controles de capital incrementa la probabilidad de que el déficit de cuenta corriente se revierta.

Las probabilidades parecen ajustarse a los datos de la muestra. Para el periodo 2013-2017, las estimaciones no muestran un incremento en la probabilidad de reversión de cuenta corriente para Costa Rica. Sin embargo, las estimaciones fuera de muestra descansan en supuestos que están sujetos a modificaciones, por tanto, deberán ser actualizadas conforme se tenga mejor información.

Los resultados no varían de manera importante al realizar las estimaciones considerando que la variable duración se registra en la base de datos como una variable discreta. Los efectos de las variables sobre la probabilidad de reversión se mantienen, $y$ aunque algunas variables pierden 
significancia estadística, las estimaciones de probabilidad están muy correlacionadas y sugieren los mismos resultados que el caso de variable continua.

Los modelos estimados pueden ser utilizados por parte de las autoridades para medir el riesgo de correcciones en la cuenta corriente bajo diversos escenarios y promover una discusión para reducir estos riesgos y así cumplir con el objetivo de mantener la estabilidad macroeconómica nacional.

El uso de la metodología de análisis de supervivencia con datos de panel para estimar la probabilidad de corrección de cuenta corriente, es poco empleado por la literatura, por lo cual futuros estudios pueden ahondar más sobre otras variables que puedan tener algún efecto y mejorar la capacidad predicativa de los modelos aquí presentados.

\section{REFERENCIAS}

Allison, P. (2010). Survival Analysis. En G. Hancock. \& R. Mueller, (Ed.). The Reviewer's Guide to Quantitative Methods in the Social Sciences, (pp 413-424). New York: Routledge.

Bugamelli, M. \& Paternò, F. (2009). Do workers' remittances reduce the probability of current account reversals?. World Development, 37(12), 1821-1838. http://dx.doi.org/10.1016/j.worlddev.2009.04.002

Cameron, A. C. y Trivedi, P. (2009). Microeconometrics: Methods and Applications. New York: Cambridge University Press.

Chinn, M. D. y Ito, H. (2008). A new measure of financial openness. Journal of Comparative Policy Analysis, 10(3), 309-322. http://dx.doi.org/10.1080/13876980802231123

Calvo G. (Noviembre, 1998). Capital Flows and Capital-Market Crises: The Simple Economics of Sudden Stops. Journal of Applied Economics, 1(1), 35-54. Recuperado de https://www.ucema. edu.ar/publicaciones/download/volume1/calvo.pdf

De Sousa, J. (2012). The currency union effect on trade is decreasing over time. Economics Letters, 117(3), 917-920. http://dx.doi.org/10.1016/j.econlet.2012.07.009

Edwards, S. (2004). Financial openness, sudden stops and current account reversals. (Working Paper 10277), National Bureau of Economic Research. http://dx.doi.org/ 10.3386/w10277

Edwards, S. (Marzo, 2005). Capital controls, sudden stops and current account reversals. (Working Paper 11170), National Bureau of Economic Research. http://dx.doi.org/10.3386/w11170

Eichengreen, B. y Adalet, M. (2005). Current account reversals: Always a problem?. (Working Paper 11634), National Bureau of Economic Research. http://dx.doi.org/10.3386/w11634

Ferretti, G. M. M. y Razin, A. (2000). Current Account Reversals and Currency Crises, Empirical Regularities. En P. Krugman (Ed.), Currency Crises, (pp. 285-326). Chicago: University of Chicago Press. http://dx.doi.org/10.3386/w6620

Fouré, J., Bénassy-Quéré, A., \& Fontagné, L. (2010). The world economy in 2050: a tentative picture. (Working Papers 2010-27), CEPII research center. Recuperado de http://www.cepii.fr/CEPII/ en/publications/wp/abstract.asp?NoDoc $=2800$

Greene, W. (2012). Econometric Analysis. New York: Pearson.

Golder, M. (2013). Discrete time duration models: BTSCS. introduction to duration models. Class notes. Department of Political Science at Pennsylvania State University. Recuperado de http:// jdemeritt.weebly.com/uploads/2/2/7/7/22771764/maths1.pdf

Gutierrez, R. G. (2002). Parametric frailty and shared frailty survival models. The Stata Journal, 2(1), 22-44. Recuperado de http://www.stata-journal.com/sjpdf.html?articlenum=st0006

Hess, W. y Persson, M. (2010). The duration of trade revisited: Continuous-time vs. discrete-time hazards. (Working Paper Series 829), Research Institute of Industrial Economics. 
Iceland, J. (1997). The dynamics of poverty spells and issues of left-censoring. (PSC Research Report No. 97-378). Population Studies Center. University of Michigan.

Fondo Monetario Internacional (2007). World Economic Outlook, April 2007. International Monetary Fund. Recuperado de https://www.imf.org/external/pubs/ft/weo/2007/01/index.htm

Fondo Monetario Internacional (2008). World Economic Outlook, October 2008. International Monetary Fund. Recuperado de https://www.imf.org/external/pubs/ft/weo/2008/02/pdf/text.pdf

Jauregui, J. M. (2006). The persistence of the current account surplus following an external sector crisis. Recuperado de http://resources.rybinski.eu/resources/sendFile:199e018a-be5a-11de-85be001b24eff4d8:1

Jenkins, S. (2004). Survival analysis. (Documento inédito), Institute for Social and Economic Research, University of Essex, Colchester, UK.

Kintanar, K. A. U. (2009). On the duration of large current account imbalances. (tesis doctoral, Department of Economics. Fordham University). Recuperado de http://fordham.bepress.com/ dissertations/AAI3384640/

Lancaster, T. (1992). The Econometric Analysis of Transition Data. Econometric Society Monographs. Cambridge University Press.

Liu, X. (2012). Survival Analysis: Models and Applications. Wiley.

http://dx.doi.org/10.1002/9781118307656

Marshall, Monty, G., G. T. R. y Jaggers, K. (2013). Polity тм IV Project. Political Regime Characteristics and Transitions, 1800-2012. Center for Systemic Peace.

Milesi-Ferrett, G. M. \& Razin, A. (1997). Sharp reductions in current account deficits: An empirical analysis. (NBER Working Papers 6310), National Bureau of Economic Research. Recuperado de https://www.imf.org/external/pubs/ft/wp/wp97168.pdf

Monge C. y Torres C. (2010). Costa Rica: Estimación del déficit en Cuenta Corriente coherente con flujos de capital de largo plazo sostenibles. Periodo 1999-2009. Banco Central de Costa Rica. DEC-DIE-DT-05-2010. Recuperado de http://www.bccr.fi.cr/investigacioneseconomicas/ politicacambiariaysectorexterno/Deficit_en_cuenta_corriente_coherente_con_flujos_de_ capital_de_largo_plazo.pdf

Obstfeld, M. \& Rogoff, K. (2000). The six major puzzles in international macroeconomics: Is there a common cause?. Working Paper 7777, National Bureau of Economic Research. http://dx.doi.org/10.3386/w7777

Programa Estado de la Nación (2012). Decimoctavo Informe Estado de la Nación en Desarrollo Humano Sostenible. San José, Estado de la Nación.

Recuperado de http://estadonacion.or.cr/index-en/informe-xviii-estado-nacion

Rose, A. K., Supaat, S. y Braude, J. (Mayo, 2009). Fertility and the real exchange rate. Canadian Journal of Economics, 42(2), 496-518. http://dx.doi.org/10.1111/j.1540-5982.2009.01517.x

Singer, J. D. y Willet, J. B. (1993). It's about time: using discrete-time survival analysis to study duration and the timing of events. Journal of Educational Statistics, 18(2), 155-195.

http://dx.doi.org/10.3102/10769986018002155

StataCorp (2013). Stata Survival Analysis and epidemiological tables. Reference Manual. Release 13. Recuperado de http://www.stata.com/manuals13/st.pdf

United Nations Conference on Trade and Development (2008). Trade and development report. Recuperado de http://unctad.org/en/Pages/Publications/TradeandDevelopmentReport.aspx 


\section{ANEXOS}

\section{Anexo 1: Evolución de la Cuenta Corriente}

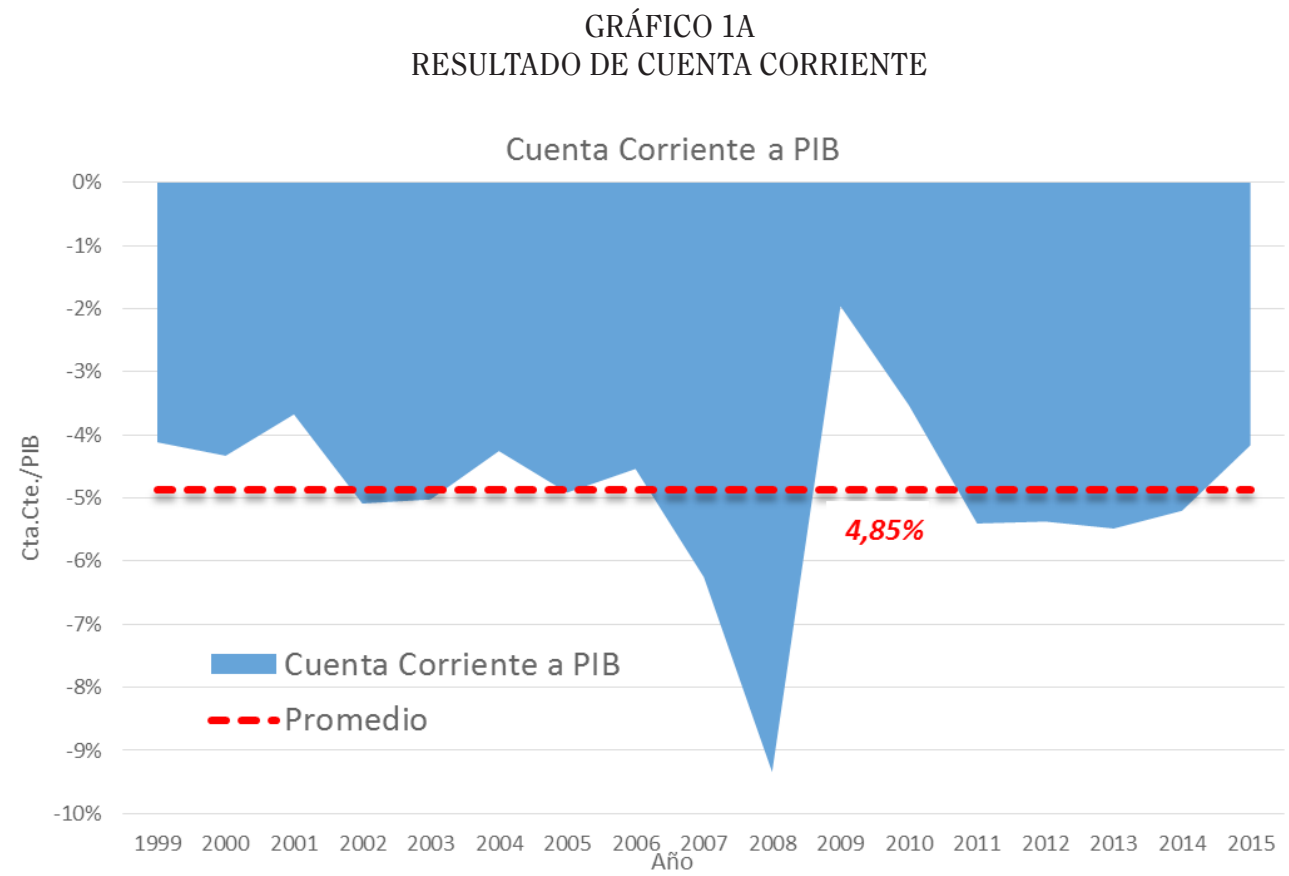

Fuente: Elaboración propia, con datos del BCCR.

Anexo 2: Funciones de sobreviviencia

FIGURA 1A

FUNCIÓN DE SOBREVIVENCIA PARA EL CRECIMIENTO DE LA PTF A 5 AÑOS

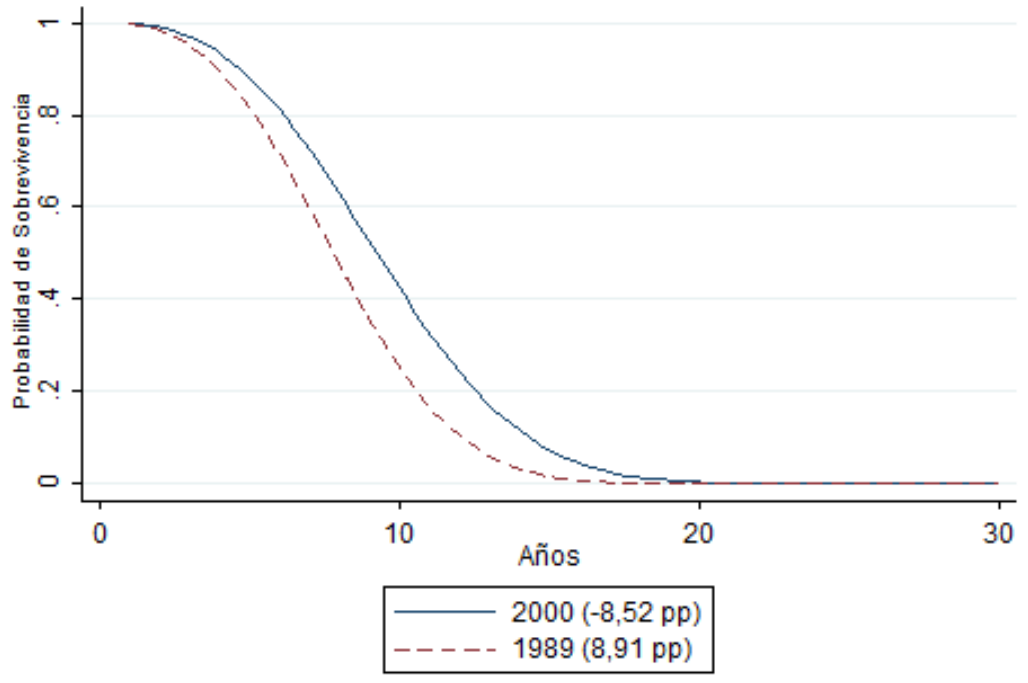

Fuente: Elaboración propia. 
FIGURA 2A

FUNCIÓN DE SOBREVIVENCIA PARA LA TASA DE DEPENDENCIA

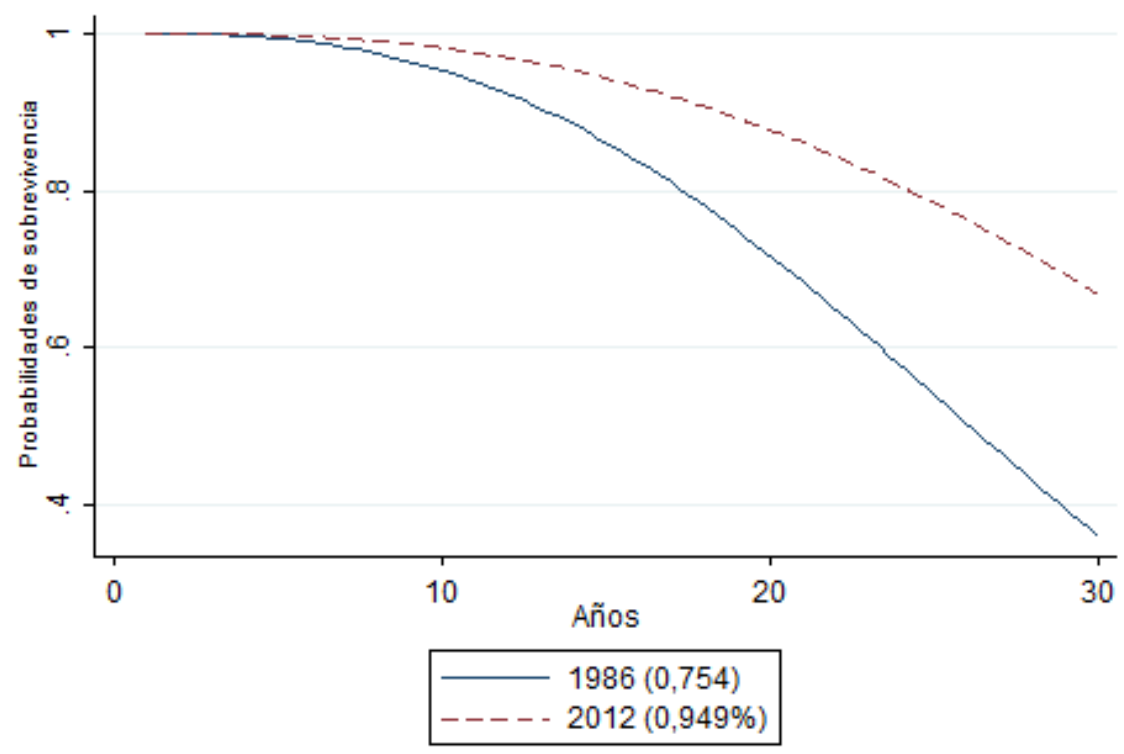

Fuente: Elaboración propia.

FIGURA 3A

FUNCIÓN DE SOBREVIVENCIA PARA LOS ACUERDOS COMERCIALES

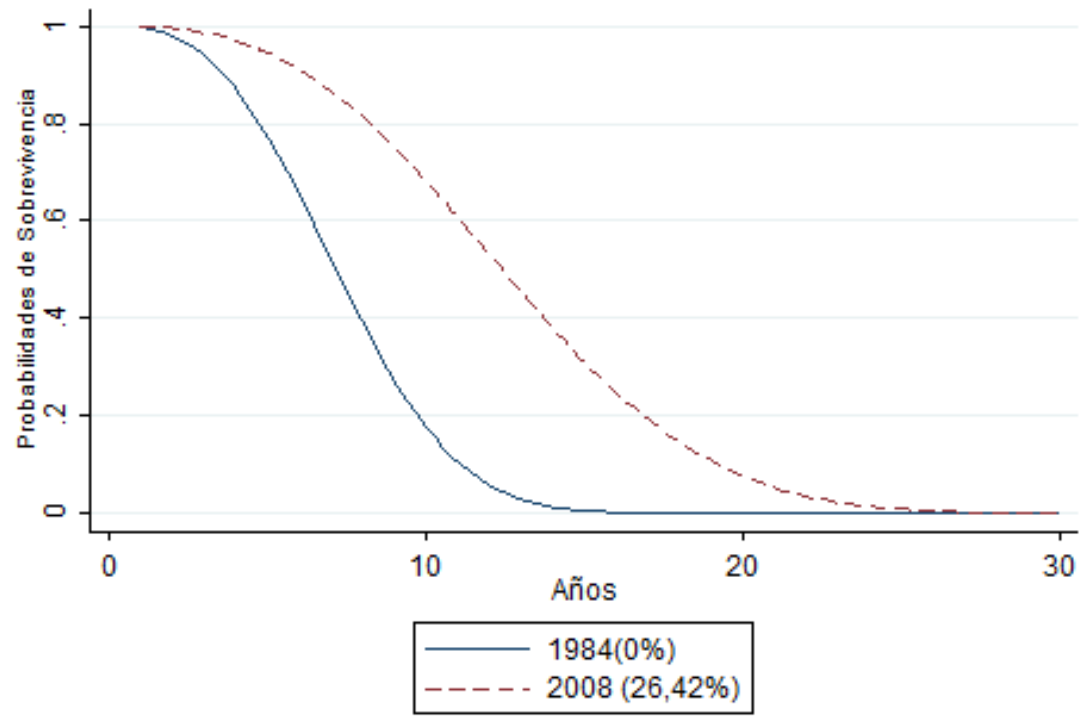

Fuente: Elaboración propia. 
FIGURA 4A

FUNCIÓN DE SOBREVIVENCIA PARA LOS ACUERDOS COMERCIALES

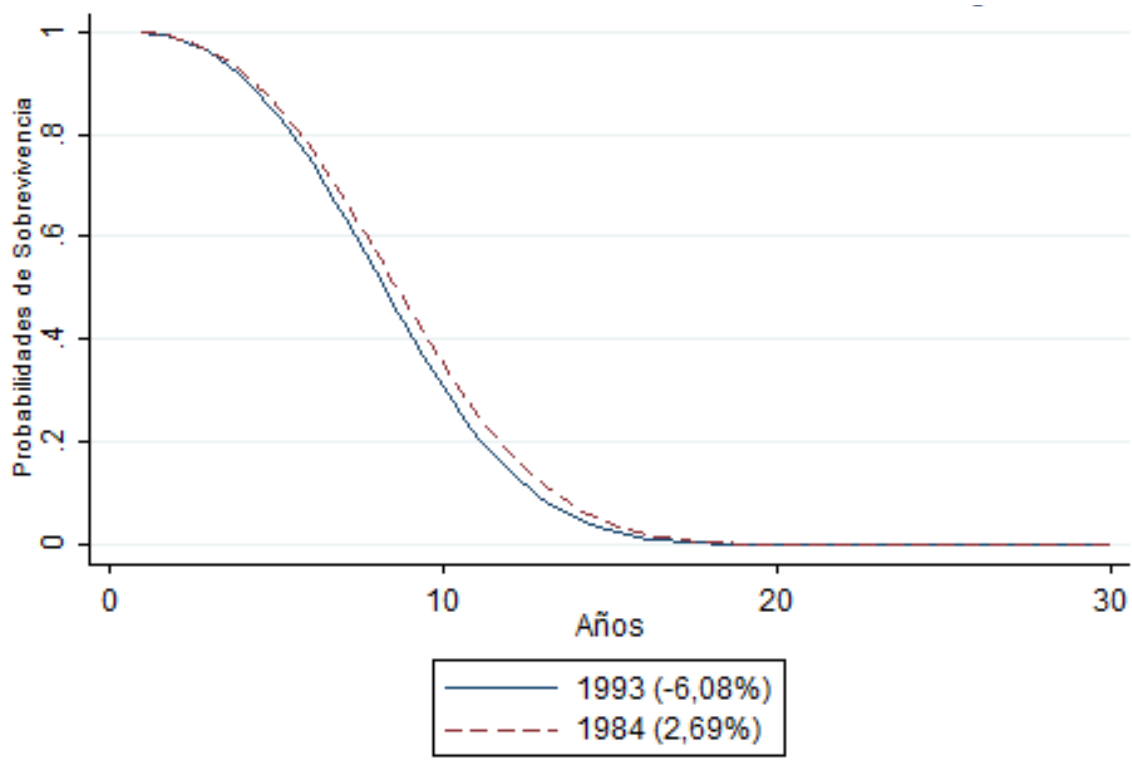

Fuente: Elaboración propia.

FIGURA 5A

FUNCIÓN DE SOBREVIVENCIA PARA LOS ACUERDOS COMERCIALES

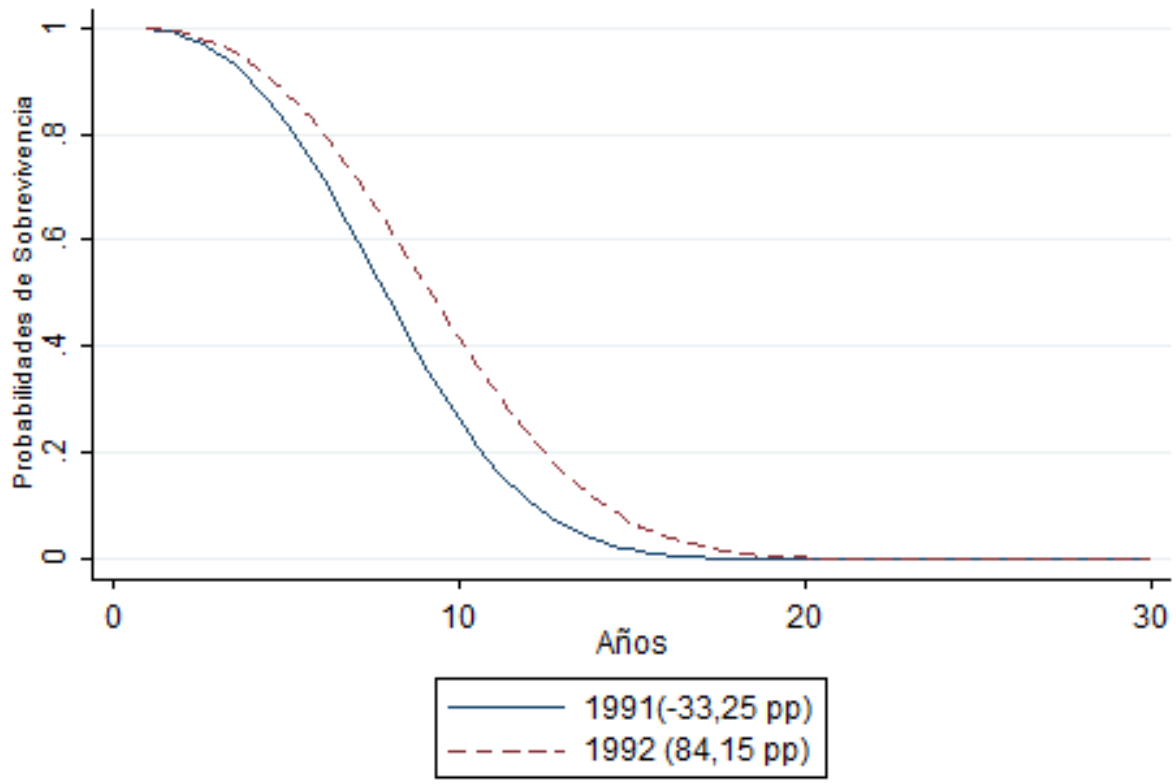

Fuente: Elaboración propia. 
FIGURA 6A

FUNCIÓN DE SOBREVIVENCIA PARA CONTROL DE CAPITALES

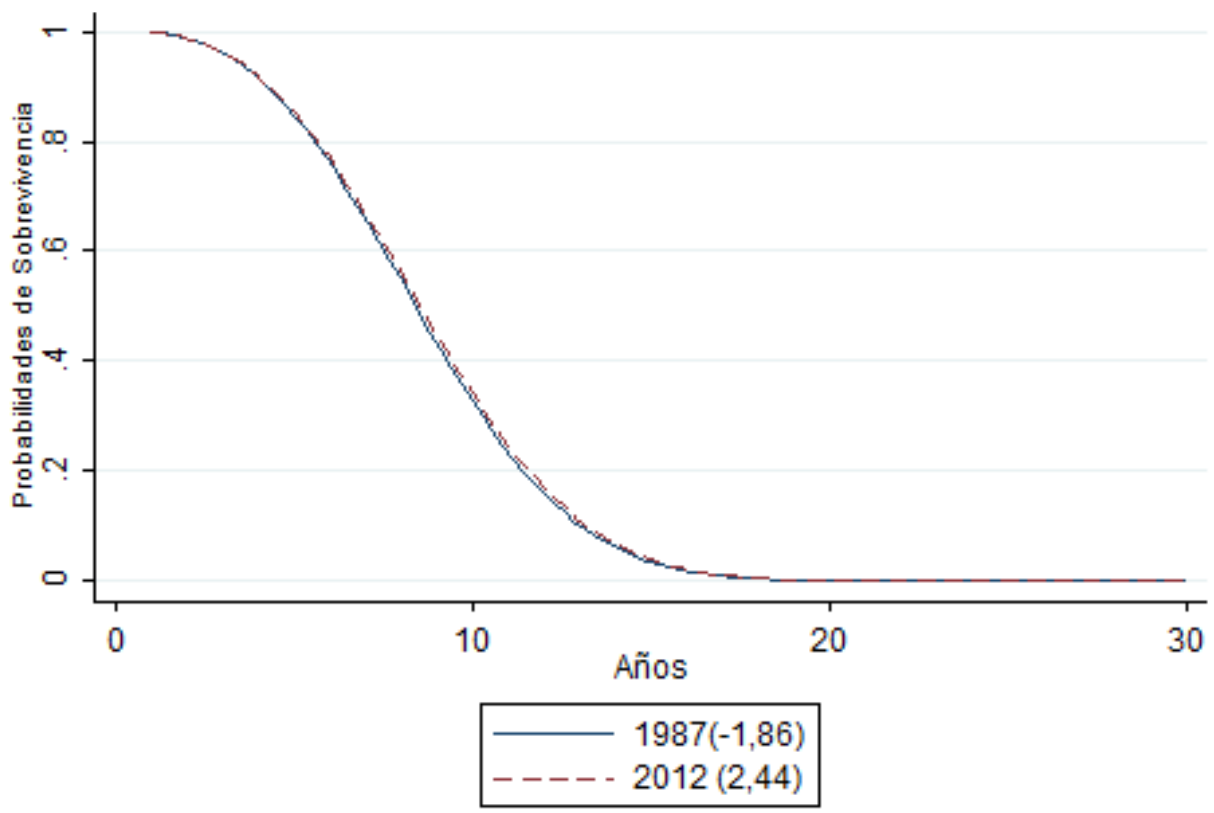

Fuente: Elaboración propia. 
Anexo 3. Países incluidos en la muestra para el caso de reversiones de al menos 2 puntos porcentuales del PIB.

\begin{tabular}{|c|c|c|c|c|c|}
\hline - & Albania & - & Guinea & $\bullet$ & Portugal \\
\hline 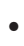 & Alemania & - & Guinea-Bissau & $\bullet$ & Qatar \\
\hline$\bullet$ & Angola & - & Guyana & • & Reino Unido \\
\hline - & Arabia Saudí & - & Haití & . & República Árabe Siria \\
\hline • & Argelia & - & Honduras & . & República Centroamericana \\
\hline . & Argentina & - & Hungría & . & República Checa \\
\hline - & Armenia & - & India & . & República Dominicana \\
\hline - & Austria & - & Indonesia & . & Ruanda \\
\hline . & Bahréin & - & Irlanda & . & Senegal \\
\hline • & Bélgica & - & Islas Salomón & . & Sierra Leone \\
\hline • & Benín & - & Israel & . & Singapur \\
\hline • & Bielorrusia & - & Italia & . & Sri Lanka \\
\hline • & Bolivia & - & Jordán & . & Suazilandia \\
\hline • & Brasil & - & Kazakstán & . & Sudáfrica \\
\hline • & Bulgaria & - & Kenia & . & Sudan \\
\hline • & Burkina Faso & - & Kuwait & . & Suiza \\
\hline • & Burundi & - & Lebanon & . & Surinam \\
\hline - & Bután & - & Lesoto & . & Tailandia \\
\hline . & Cabo Verde & - & Letonia & . & Tanzania \\
\hline • & Camboya & - & Lituania & . & Tayikistán \\
\hline • & Cameron & - & Madagascar & . & Togo \\
\hline • & Canadá & - & Malawi & . & Trinidad and Tobago \\
\hline . & Chad & - & Malaysia & . & Túnez \\
\hline - & Chile & - & Mali & . & Turquía \\
\hline • & China & - & Mauricio & . & Ucrania \\
\hline • & Colombia & - & Mauritania & . & Uganda \\
\hline • & Costa de Marfil & - & México & . & Uruguay \\
\hline • & Costa Rica & - & Moldavia & . & Venezuela, RB \\
\hline • & Dinamarca & - & Mongolia & . & Yibuti \\
\hline • & Egipto & - & Mozambique & . & Zambia \\
\hline • & Eslovaquia & - & Nepal & & \\
\hline • & España & - & Nicaragua & & \\
\hline - & Estonia & - & Níger & & \\
\hline • & Etiopia & - & Nigeria & & \\
\hline - & Filipinas & - & Noruega & & \\
\hline - & Finlandia & - & Nueva Zelanda & & \\
\hline - & Fiyi & - & Omán & & \\
\hline • & Gabón & - & Pakistán & & \\
\hline • & Gambia & - & Panamá & & \\
\hline • & Georgia & - & Papúa Nueva & & \\
\hline • & Ghana & - & Paraguay & & \\
\hline - & Grecia & - & Perú & & \\
\hline - & Guatemala & - & Polonia & & \\
\hline
\end{tabular}




\section{Anexo 4. Países incluidos en la muestra para el caso de reversiones de al menos 5 pun- tos porcentuales del PIB.}

\begin{tabular}{|c|c|c|c|c|c|}
\hline & Albania & - & Guyana & - & Omán \\
\hline & Angola & - & Haití & - & Panamá \\
\hline & Arabia Saudí & - & Honduras & - & Papúa Nueva Guinea \\
\hline & Argelia & $\bullet$ & Hungría & • & Paraguay \\
\hline & Argentina & $\bullet$ & Indonesia & - & Perú \\
\hline & Armenia & $\bullet$ & Islas Salomón & - & Polonia \\
\hline & Bahréin & $\bullet$ & Israel & - & Qatar \\
\hline & Benín & $\bullet$ & Jordán & $\bullet$ & República Centroamericana \\
\hline & Bielorrusia & $\bullet$ & Kazakstán & $\bullet$ & República Dominicana \\
\hline & Bolivia & $\bullet$ & Kenia & $\bullet$ & Ruanda \\
\hline & Bulgaria & $\bullet$ & Kuwait & $\bullet$ & Senegal \\
\hline & Burkina Faso & $\bullet$ & Lebanon & $\bullet$ & Sierra Leone \\
\hline & Burundi & $\bullet$ & Lesoto & $\bullet$ & Singapur \\
\hline & Bután & $\bullet$ & Letonia & 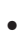 & Sri Lanka \\
\hline & Cabo Verde & $\bullet$ & Lituania & 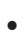 & Suazilandia \\
\hline & Camboya & • & Madagascar & 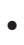 & Sudáfrica \\
\hline & Cameron & • & Malawi & 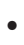 & Sudan \\
\hline & Chile & b & Malaysia & 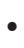 & Surinam \\
\hline & Colombia & • & Mali & 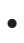 & Tailandia \\
\hline & Costa de Marfil & $\bullet$ & Mauricio & 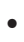 & Tanzania \\
\hline & Costa Rica & b & Mauritania & $\bullet$ & Tayikistán \\
\hline & Egipto & • & México & 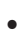 & Togo \\
\hline & Estonia & • & Moldavia & $\bullet$ & Trinidad and Tobago \\
\hline & Filipinas & • & Mongolia & $\bullet$ & Túnez \\
\hline & Fiyi & 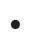 & Mozambique & $\bullet$ & Turquía \\
\hline & Gabón & $\bullet$ & Nicaragua & $\bullet$ & Ucrania \\
\hline & Gambia & $\bullet$ & Níger & 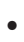 & Uganda \\
\hline & Georgia & $\bullet$ & Nigeria & $\bullet$ & Uruguay \\
\hline & Ghana & $\bullet$ & Noruega & 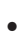 & Venezuela, RB \\
\hline & Guinea-Bissau & $\bullet$ & Nueva Zelanda & & Yibuti \\
\hline & & & & $\bullet$ & Zambia \\
\hline
\end{tabular}

\section{Anexo 5. Clasificación de las regiones.}

$\begin{array}{llll}\text { - } & \text { África Central } & - & \text { Asia Sudoriental } \\ \text { - } & \text { África del Norte } & - & \text { Australia/Nueva Zelanda } \\ \text { - } & \text { África del Sur } & - & \text { Caribe } \\ \text { - } & \text { África Occidental } & - & \text { Europa del Este } \\ \text { - } & \text { África Oriental } & - & \text { Europa del Norte } \\ \text { - } & \text { América Central } & - & \text { Europa del Oeste } \\ \text { - } & \text { América del Norte } & - & \text { Europa meridional } \\ \text { - } & \text { América del Sur } & - & \text { Melanesia } \\ \text { - } & \text { Asia Central } & - & \text { Micronesia } \\ \text { - } & \text { Asia del Sur } & - & \text { Occidental Asia } \\ \text { - } & \text { Asia Occidental } & - & \text { Polinesia }\end{array}$

